\title{
Quasi-particle description for the transport through a small interacting system
}

\author{
Akira Oguri \\ Department of Material Science, Faculty of Science, Osaka City University, \\ Sumiyoshi-ku, Osaka 558-8585, Japan
}

(October 26, 2018)

\begin{abstract}
We study effects of electron correlation on the transport through a small interacting system connected to reservoirs using an effective Hamiltonian which describes the free quasi-particles of a Fermi liquid. The effective Hamiltonian is defined microscopically with the value of the self-energy at $\omega=0$. Specifically, we apply the method to a Hubbard chain of finite size $N(=1,2,3, \ldots)$, and calculate the self-energy within the second order in $U$ in the electron-hole symmetric case. When the couplings between the chain and the reservoirs on the left and right are small, the conductance for even $N$ decreases with increasing $N$ showing a tendency toward a Mott-Hubbard insulator. This is caused by the off-diagonal element of the self-energy, and this behavior is qualitatively different from that in the special case examined in the previous work. We also study the effects of the asymmetry in the two couplings. While the perfect transmission due to the Kondo resonance occurs for any odd $N$ in the symmetric coupling, the conductance for odd $N$ decreases with increasing $N$ in the case of the asymmetric coupling.
\end{abstract}

PACS numbers: 72.10.-d, 72.10.Bg, 73.40.-c 


\section{INTRODUCTION}

Effects of the inter-electron interaction on the transport through small interacting systems have been a subject of current interest. For instance, the Kondo effect expected to be observed in a quantum dot目 has been studied intensively from both theoretical国 and experimental sides 6 Also, the Tomonaga-Luttinger behavior expected in one-dimensional systems 9.10 has been investigated in a quantum wire.11

For studying the quantum transport of small systems theoretically, a formulation which is able to treat both the interaction and interference effects in a unified way is necessary. Meir and Wingreen 12 have presented one such framework using the nonequilibrium Keldysh formalism.13,14 Especially, Eq. (6) of Ref. 12 is a general formula for the total current, and is available for various systems. While the quantum transport theory for nonequilibrium states is important to understand the physics in small systems, there are some ambiguities for determining the stationary state. So, it seems to be still meaningful to describe a formulation based on the linear response theory. In the Kubo formalism, the conductance for non-interacting electrons at $T=0$ can be expressed in terms of the Green's function at Fermi energy. 15 This is also true for interacting electrons if the ground state is a Fermi liquid 18,19 The purpose of this paper is to apply a quasi-particle description of a Fermi liquid to the conductance of a small interacting systems. At $T=0$, an effective Hamiltonian for free quasi-particles can be defined microscopically based on the perturbation theory, which reproduces the value of the Green's function at $\omega=0$. This kind of an effective Hamiltonian has been introduced, for instance, for the single Anderson impurity and systems with a translational invariance.20 One of the characteristics of the small interacting system is that the self-energy has off-diagonal elements. This is because generally the system consists of a number of resonant states and has no translational invariance.

Specifically, we apply the method to a Hubbard chain of finite size $N(=1,2,3, \ldots)$ connected to non-interacting leads. This system can be regarded as a model for a MottHubbard insulator of nanometer scale. Also, for small $N$, the system can regarded as a model 
for a series of quantum dots, which has been examined by advanced numerical methods such as the numerical renormalization group21 and quantum Monte Carlo methods.19,2223 It may also be considered as a model for a quantum wire, which has been studied with the bosonization approach take into account the Umklapp scattering.24 28 We calculate all the elements of a $N \times N$ matrix self-energy within the second order in $U$ in the electronhole symmetric case. The unperturbed Hamiltonian is taken to be connected including the coupling between the Hubbard chain and leads in it as that in the case of the Anderson impurity.29 In the previous paper, 30 we have reported the results obtained in a special case $v_{L}=v_{R}=t$, where $v_{L}\left(v_{R}\right)$ is the tunneling matrix element between the chain and the left (right) lead, and $t$ is the hopping matrix element of the chain. In this case, one can obtain the self-energy analytically owing to the property that the non-interacting system has the translational invariance. However, this particular feature is realized only in this case, and some behaviors seeing in the results seem to depend on it: the reduction of the conductance for even $N$ is proportional to $U^{4}$ for small $U$, and in the limit of large even $N$ the conductance tends to be finite.

In the present work, we examine the dependence of the conductance on the coupling $v_{L}$ and $v_{R}$ calculating the self-energy numerically. In contrast to the special case mentioned above, the results obtained in weak coupling cases $v_{L}, v_{R}<t$ show quite different behaviors: the reduction of the conductance for even $N$ is proportional to $U^{2}$ for small $U$, and the conductance tends to zero for large even $N$. Physically, the difference between the weak coupling cases $v_{L}, v_{R}<t$ and the special case $v_{L}=v_{R}=t$ is caused by whether $N$ revels in the chain form distinguishable resonant states or not. The effect of the interaction is enhanced for the weak coupling cases. It is also shown generally beyond the second-order perturbation that the perfect transmission occurs for odd $N$ when the system has both the inversion symmetry $v_{L}=v_{R}(\equiv v)$ and the electron-hole symmetry. This is due to Kondo resonance and independent of the values of $v$ and $U$. We also examine the effects of the asymmetry in the coupling $v_{L} \neq v_{R}$, which disturbs the perfect transmission. We note that preliminary results were reported in a proceedings. 31 
In Sec. [1, we introduce the effective Hamiltonian, and give the interpretation of the dc conductance and total charge displacement in terms of the free quasi-particles. In Sec. [IT, we show some properties of the quasi-particles in the electron-hole symmetric case without specifying details of the model. In Sec. $\mathbb{\nabla}$, we apply the method to the Hubbard chain of finite size, and present the results obtained with the second-order perturbation theory. Summary is given in Sec. $\mathrm{Q}$.

\section{EFFECTIVE HAMILTONIAN AND GROUND-STATE PROPERTIES}

In this section, we introduce an effective Hamiltonian for the free quasi-particles based on the perturbation theory in the inter-electron interaction. In this context, the dc conductance and total charge displacement at $T=0$ are described by the scattering coefficients of the free quasi-particles. In what follows, we will discuss the formulation assuming the single-mode leads for simplicity. The formulation can be generalized to the multi-mode leads.

We start with a system which consists of three regions; a finite central region $(C)$ and the two non-interacting leads on the left $(L)$ and the right $(R)$. The central region consists of $N$ resonant levels, and the interaction is switched on only for the electrons in this region. We assume that the two leads consist of infinite degrees of freedom, respectively, and are connected to the central region by the mixing matrix elements $v_{L}$ and $v_{R}$ as illustrated in Fig. 1. The complete Hamiltonian is given by

$$
\begin{aligned}
\mathcal{H} & =\mathcal{H}_{L}+\mathcal{H}_{R}+\mathcal{H}_{C}^{0}+\mathcal{H}_{C}^{i n t}+\mathcal{H}_{m i x}, \\
\mathcal{H}_{L} & =\sum_{i j \in L} \sum_{\sigma}\left(-t_{i j}^{L}-\mu \delta_{i j}\right) c_{i \sigma}^{\dagger} c_{j \sigma}, \\
\mathcal{H}_{R} & =\sum_{i j \in R} \sum_{\sigma}\left(-t_{i j}^{R}-\mu \delta_{i j}\right) c_{i \sigma}^{\dagger} c_{j \sigma}, \\
\mathcal{H}_{C}^{0} & =\sum_{i j \in C} \sum_{\sigma}\left(-t_{i j}^{C}-\mu \delta_{i j}\right) c_{i \sigma}^{\dagger} c_{j \sigma}, \\
\mathcal{H}_{C}^{i n t} & =\frac{1}{2} \sum_{\{j\} \in C} \sum_{\sigma \sigma^{\prime}} U_{j_{4} j_{3} ; j_{2} j_{1}} c_{j_{4} \sigma}^{\dagger} c_{j_{3} \sigma^{\prime}}^{\dagger} c_{j_{2} \sigma^{\prime}} c_{j_{1} \sigma},
\end{aligned}
$$




$$
\mathcal{H}_{m i x}=-\sum_{\sigma} v_{L}\left(c_{1 \sigma}^{\dagger} c_{0 \sigma}+c_{0 \sigma}^{\dagger} c_{1 \sigma}\right)-\sum_{\sigma} v_{R}\left(c_{N+1 \sigma}^{\dagger} c_{N \sigma}+c_{N \sigma}^{\dagger} c_{N+1 \sigma}\right)
$$

Here $c_{j \sigma}^{\dagger}\left(c_{j \sigma}\right)$ creates (destroys) an electron with spin $\sigma$ at site $j$, and $\mu$ is the chemical potential. $t_{i j}^{L}, t_{i j}^{R}$, and $t_{i j}^{C}$ are the intra-region hopping matrix elements in each regions $L, R$, and $C$, respectively. The coupling between the central region and two leads are described by the mixing matrix element $\mathcal{H}_{m i x}$. We assign the labels $1,2, \ldots, N$ to the sites in the central region. Specifically, we assign the labels 1 and $N$ to the sites at the interface on the left and right, respectively. Correspondingly, the label 0 and $N+1$ are assigned to the sites at the lead-side of the interface on the left and right, respectively [see Fig. 1]. The inter-electron interaction $U_{j_{4} j_{3} ; j_{2} j_{1}}$ is switched on in the central region, and it has the time reversal symmetry: $U_{43 ; 21}$ is real and $U_{43 ; 21}=U_{34 ; 12}=U_{12 ; 34}=U_{42 ; 31}=U_{13 ; 24}$. We take all the hopping matrix elements to be real, and will be using units $\hbar=1$ unless otherwise noted.

In the limit $N=1$, the model reduces to a single Anderson impurity in which the perturbation expansion is valid for all values of $U .22$ Our basic idea is to apply the perturbation theory in $\mathcal{H}_{C}^{\text {int }}$ following Yamada and Yosida 29 regarding the central region as one big impurity. Therefore, we take the unperturbed part of the Hamiltonian $\mathcal{H}^{(0)}$ to be connected by including the mixing term $\mathcal{H}_{\text {mix }}$ in it;

$$
\mathcal{H}^{(0)}=\mathcal{H}_{L}+\mathcal{H}_{R}+\mathcal{H}_{C}^{0}+\mathcal{H}_{\text {mix }}
$$

Our working hypothesis is that the ground state is changed continuously against the adiabatic switching-on of the interaction $\mathcal{H}_{C}^{\text {int }}$. The perturbation expansion can be done using the single-particle Green's function

$$
G_{j j^{\prime}}\left(i \varepsilon_{l}\right)=-\int_{0}^{\beta} d \tau\left\langle T_{\tau} c_{j \sigma}(\tau) c_{j^{\prime} \sigma}^{\dagger}(0)\right\rangle e^{i \varepsilon_{l} \tau}
$$

where $\beta=1 / T, \varepsilon_{l}=(2 l+1) \pi / \beta, c_{j \sigma}(\tau)=e^{\tau \mathcal{H}} c_{j \sigma} e^{-\tau \mathcal{H}}$, and $\langle\cdots\rangle$ denotes the thermal average $\operatorname{Tr}\left[e^{-\beta \mathcal{H}} \ldots\right] / \operatorname{Tr} e^{-\beta \mathcal{H}}$. The spin index has been omitted from the left-hand side of Eq. (8) assuming the expectation value to be independent of whether spin is up or down. 
Since the interaction is switched on only for the electrons in the central region, the Dyson equation is written as

$$
G_{i j}(z)=G_{i j}^{(0)}(z)+\sum_{l l^{\prime} \in C} G_{i l}^{(0)}(z) \Sigma_{l l^{\prime}}(z) G_{l^{\prime} j}(z)
$$

Here $G_{i j}^{(0)}(z)$ is the unperturbed Green's function corresponding to $\mathcal{H}^{(0)}$. The summations with respect to $l$ and $l^{\prime}$ run over the sites in the central region, and $\Sigma_{l l^{\prime}}(z)$ is the self-energy correction due to $\mathcal{H}_{C}^{i n t}$. Note that $G_{i j}(z)=G_{j i}(z)$ and $\Sigma_{i j}(z)=\Sigma_{j i}(z)$ because of the time reversal symmetry of $\mathcal{H}$. In what follows, we will treat $z$ as a complex variable, i.e., $G_{i j}(z)$ is the analytic continuation of $G_{i j}\left(i \varepsilon_{l}\right)$. When the perturbation expansion is valid, the singleparticle excitation at the Fermi energy $z=i 0^{+}$does not decay at $T=0$ owing to a property of the Fermi liquid目

$$
\operatorname{Im} \Sigma_{i j}^{+}(0)=0
$$

Here the superscript $(+)$ is the label for a retarded function: we will use a notation $\Sigma_{i j}^{ \pm}(\omega) \equiv$ $\Sigma_{i j}\left(\omega \pm i 0^{+}\right) 33$ Due to this property, the Dyson equation Eq. (9) for $\omega=0$ can be mapped on to a scattering problem of the free quasi-particles described by the effective Hamiltonian

$$
\begin{aligned}
\widetilde{\mathcal{H}}_{q p} & =\mathcal{H}_{L}+\mathcal{H}_{R}+\widetilde{\mathcal{H}}_{C}+\mathcal{H}_{m i x}, \\
\widetilde{\mathcal{H}}_{C} & =\sum_{i j \in C} \sum_{\sigma}\left(-\widetilde{t}_{i j}^{C}-\mu \delta_{i j}\right) c_{i \sigma}^{\dagger} c_{j \sigma}, \\
-\widetilde{t}_{i j}^{C} & =-t_{i j}^{C}+\operatorname{Re} \Sigma_{i j}^{+}(0) .
\end{aligned}
$$

The value of the Green's function corresponding to $\widetilde{\mathcal{H}}_{q p}$ and the one corresponding $\mathcal{H}$ are the same at $T=0, \omega=0$.

We now consider the conductance with the Kubo formalism. If the ground state is a Fermi liquid, the contributions of the vertex corrections for the dc conductance vanish at $T=0.19$ Therefore, the dc conductance at $T=0$ is written in terms of the Green's function at $\omega=0$ also for interacting electrons; 4

$$
g_{N}=\frac{2 e^{2}}{h} 4 \Gamma_{R}(0) G_{N 1}^{+}(0) \Gamma_{L}(0) G_{1 N}^{-}(0)
$$


Here $\Gamma_{L}(\omega)=-\operatorname{Im}\left[v_{L}^{2} g_{L}^{+}(\omega)\right]$, and $\Gamma_{R}(\omega)=-\operatorname{Im}\left[v_{R}^{2} g_{R}^{+}(\omega)\right] . g_{L}^{+}\left(g_{R}^{+}\right)$is the Green's function at the interface $i=0(i=N+1)$ of the isolated lead, and it is determined by $\mathcal{H}_{L}\left(\mathcal{H}_{R}\right)$. Note that Eq. (14) is also expressed in terms of the transmission probability of the free quasi-particles, $|\widetilde{t}(0)|^{2}=4 \Gamma_{R}(0) G_{N 1}^{+}(0) \Gamma_{L}(0) G_{1 N}^{-}(0)$. 14.,55.36 Correspondingly, the reflection probability is given by $|\widetilde{r}(0)|^{2}=\left|1-2 i \Gamma_{L}(0) G_{11}^{+}(0)\right|^{2}=\left|1-2 i \Gamma_{R}(0) G_{N N}^{+}(0)\right|^{2}$, and the unitarity $|\widetilde{t}(0)|^{2}+|\widetilde{r}(0)|^{2}=1$ is preserved owing to the property Eq. (10) [see also (22)]. Another quantity which can be related to the scattering coefficients is the displacement of the total charge 33.37

$$
\Delta N_{t o t}=\sum_{i \in C} \sum_{\sigma}\left\langle c_{i \sigma}^{\dagger} c_{i \sigma}\right\rangle+\sum_{i \in L} \sum_{\sigma}\left[\left\langle c_{i \sigma}^{\dagger} c_{i \sigma}\right\rangle-\left\langle c_{i \sigma}^{\dagger} c_{i \sigma}\right\rangle_{L}\right]+\sum_{i \in R} \sum_{\sigma}\left[\left\langle c_{i \sigma}^{\dagger} c_{i \sigma}\right\rangle-\left\langle c_{i \sigma}^{\dagger} c_{i \sigma}\right\rangle_{R}\right] .
$$

Here $\langle\cdots\rangle_{L}$ and $\langle\cdots\rangle_{R}$ denote the ground-state average of isolated leads described by $\mathcal{H}_{L}$ and $\mathcal{H}_{R}$, respectively. At $T=0, \Delta N_{\text {tot }}$ can be expressed in terms of the $S$-matrix for the quasi-particles, following the derivation of the Friedel sum rule by Langer and Ambegaokar,33 as

$$
\begin{aligned}
\Delta N_{\text {tot }} & =\frac{1}{\pi i} \log [\operatorname{det} \boldsymbol{S}], \\
\boldsymbol{S} & =\left[\begin{array}{ll}
1 & 0 \\
0 & 1
\end{array}\right]-2 i\left[\begin{array}{cc}
\Gamma_{L}(0) & 0 \\
0 & \Gamma_{R}(0)
\end{array}\right]\left[\begin{array}{cc}
G_{11}^{+}(0) & G_{1 N}^{+}(0) \\
G_{N 1}^{+}(0) & G_{N N}^{+}(0)
\end{array}\right] .
\end{aligned}
$$

Therefore, the conductance and charge displacement are determined by the inter- and intraboundary elements of the Green's function such as $G_{N 1}^{+}(0)$ and $G_{11}^{+}(0)$.

Next we discuss the structure of the Dyson equation Eq. (9) further in order to make the mathematical features of the perturbation theory in the presence of the reservoirs clear. For the Green's functions in the central region, Eq. (9) is written in a $N \times N$ matrix form;

$$
\{\mathcal{G}(z)\}^{-1}=\left\{\mathcal{G}^{(0)}(z)\right\}^{-1}-\Sigma(z)
$$

Here $\mathcal{G}(z)=\left\{G_{i j}(z)\right\}$ with $i j \in C$, and the inverse matrix of the unperturbed part can be expressed as $\left\{\mathcal{G}^{(0)}(z)\right\}^{-1}=z \mathbf{1}-\mathcal{H}_{C}^{0}-\mathcal{V}_{\text {mix }}(z)$ with 


$$
\begin{aligned}
& \mathcal{H}_{C}^{0}=\left[\begin{array}{cccc}
-t_{11}^{C}-\mu & -t_{12}^{C} & \cdots & \\
-t_{21}^{C} & -t_{22}^{C}-\mu & & \\
\vdots & & \ddots & \\
& & & -t_{N N}^{C}-\mu
\end{array}\right] \\
& \mathcal{V}_{\text {mix }}(z)=\left[\begin{array}{ccccc}
v_{L}^{2} g_{L}(z) & 0 & \cdots & 0 & 0 \\
0 & 0 & \cdots & 0 & 0 \\
\vdots & \vdots & \ddots & \vdots & \vdots \\
0 & 0 & \cdots & 0 & 0 \\
0 & 0 & \cdots & 0 & v_{R}^{2} g_{R}(z)
\end{array}\right] \\
& \Sigma(z)=\left[\begin{array}{cccc}
\Sigma_{11}(z) & \Sigma_{12}(z) & \cdots & \\
\Sigma_{21}(z) & \Sigma_{22}(z) & & \\
\vdots & & \ddots & \\
& & & \Sigma_{N N}(z)
\end{array}\right] \text {. }
\end{aligned}
$$

Here 1 is the $N \times N$ unit matrix. $\mathcal{V}_{\text {mix }}$ corresponds to the contribution of the mixing with the reservoirs. Especially, the two non-zero elements $v_{L}^{2} g_{L}$ and $v_{R}^{2} g_{R}$ have the finite imaginary parts corresponding to $\Gamma_{L}$ and $\Gamma_{R}$ defined just bellow Eq.(14). These imaginary parts change the discrete levels of $\mathcal{H}_{C}^{0}$ to the continuous peaks with finite level width, and thus the unperturbed part of the Green's function $\mathcal{G}^{(0)}(z)$ describes a system of $N$ resonant scatterers. Therefore, owing to the contribution of the mixing, the mathematical structure of the perturbation theory in the presence of the reservoirs becomes similar to that of the Anderson model with a number of orbits rather than the usual Hubbard model without reservoirs. In this matrix form, the quasi-particle description is summarized as follows. Due to the property $\operatorname{Im} \Sigma^{+}(0)=0$ at $T=0$, the Green's function at $\omega=0$ is written as $\left\{\mathcal{G}^{+}(0)\right\}^{-1}=\mathcal{K}-\mathcal{V}_{\text {mix }}^{+}(0)$, where $\mathcal{K}$ is the renormalized hopping matrix corresponding to $-\widetilde{\mathcal{H}}_{C}$ defined by Eq. (12);

$$
\mathcal{K}=-\left[\mathcal{H}_{C}^{0}+\operatorname{Re} \boldsymbol{\Sigma}^{+}(0)\right]
$$

Then a relation corresponding to the optical theorem can be obtained as $\mathcal{G}^{+}(0)-\mathcal{G}^{-}(0)=$ $\mathcal{G}^{+}(0)\left[\mathcal{V}_{m i x}^{+}(0)-\mathcal{V}_{m i x}^{-}(0)\right] \mathcal{G}^{-}(0)$, which certifies the unitarity of the scattering coefficients. 
Here the superscript $+(-)$ means the retarded (advanced) function.

The quasi-particle approach cannot be applied to a non-Fermi-liquid ground state where the perturbation theory with respect to the inter-electron interaction breaks down. However, in the finite interacting system connected to reservoirs as illustrated in Fig. 1, a cross over from a high temperature phase to the low temperature Fermi liquid phase is expected in many cases. This is because, due to the connection with the reservoirs, not only the energy scale corresponding to the level spacing of the isolated interacting system of size $N$ but also the level width introduced through $\Gamma_{L}$ and $\Gamma_{R}$, i.e., the imaginary part of the mixing term Eq. (20), plays a role of the cut-off. In this sense, as mentioned above, the system can be regarded as a generalized Anderson impurity with $N$ resonant states.

When the interacting region is described by an one-dimensional chain, the TomonagaLuttinger (TL) behavior 38 seems to be seen for large $N$. However, as discussed by Kane and Fisher for the finite TL model of size $L$ connected to Fermi liquid reservoirs 9 the cross over from the high temperature TL phase to the low temperature Fermi liquid phase occurs at a characteristic energy $T_{0} \simeq v_{F} / L$, where $v_{F}$ is the Fermi velocity. Since this simple discussion does not take into account various effects such as the back and Umklapp scatterings, the characteristic energy will be different depending on the details of the situations. Specifically, in the electron-hole symmetric case of the Hubbard chain examined in the following chapters, the characteristic energy depends on whether $N$ is even or odd, and it should be the MottHubbard gap $E_{G}$ or the Kondo temperature $T_{K}$. Again, due to the contribution of the mixing, the low energy excitations below the characteristic energy scale are described by the Fermi liquid as far as $N$ is finite. For even $N$, the Mott-Hubbard gap opens in the thermodynamic limit of the usual Hubbard model without reservoirs, and the corresponding tendency should be seen in the present system of finite $N$. However, there still remains a finite density of states at the Fermi energy caused by the mixing with the reservoirs, although the spectral weight in the region corresponding to the gap will decrease with increasing $N$. For odd $N$, there is an additional spectral weight at the Fermi energy in the Mott-Hubbard gap, i.e., the Kondo resonance of the width $T_{K}$. Thus, the insulator-like 
low-conductivity should be seen at the temperature range $T_{K}<T<E_{G}$, and the metallic behavior of the Fermi liquid will be seen at low temperatures $T<T_{K}$. Note that $T_{K}$ can be defined only for the open system connected to reservoirs, while $E_{G}$ is a constant defined in the thermodynamic limit of the isolated system. Furthermore, $T_{K}$ should decrease with increasing $N$, and in most of cases $T_{K}$ should vanish in the limit of $N \rightarrow \infty$. Therefore, if the limit $N \rightarrow \infty$ is taken keeping $T$ to be finite, the even-odd behavior disappears and at $T<E_{G}$ the insulating behavior meets with an intuitive picture will be seen. This limit corresponds to the thermodynamic limit of the macroscopic system. However, there is another nontrivial limit which describes the low temperature physics of the mesoscopic systems, i.e., the limit $T \rightarrow 0$ keeping $N$ to be finite. The ground state of the small system belongs to this limit, and in the case of the small Hubbard chain the even-odd behavior can be seen at low temperatures since $T_{K}$ is finite for small $N$. This kind of a mesoscopic limit is expected to be realized in quantum dots or quantum wire of nanometer size, and our aim here is to develop a Fermi-liquid theory for the mesoscopic systems.

Another possible non-Fermi-liquid state is the ground state of the multi-channel Kondo

system. 39 Shimizu, Sakai and Suzuki have shown with the numerical renormalization group approach that the non-Fermi-liquid ground state is realized also in an extended version of the Anderson model 4 So, if the parameters of the Hamiltonian Eq. (11) satisfy certain conditions, the similar situation seems to be realized.

\section{QUASI-PARTICLES IN THE ELECTRON-HOLE SYMMETRIC CASE}

The effective Hamiltonian has some notable properties in the electron-hole symmetric case where the average number of electrons in each site is unity. In this section, we provide a simplified expression of the conductance in the electron-hole symmetric case. The results Eqs. (31) and (33) will be used for the finite Hubbard chain in the next section. Especially, form Eq. (31), we can deduce quite generally that the perfect transmission occurs for odd $N$ when the system has an additional inversion symmetry. 
In the electron-hole symmetric case, the off-diagonal element of the renormalized parameter $\widetilde{t}_{i j}^{C}$ is zero when $i$ and $j$ belong to the same sublattice, i.e., $-\widetilde{t}_{i j}^{C}-\mu \delta_{i j}=0$ for $|i-j|=0,2,4, \ldots$, and $\operatorname{Re} \mathcal{V}_{m i x}^{+}(0)=0$. Thus, the matrix $\mathcal{K}$ defined by Eq. (22) has a checkered structure, and the Green's function at $T=0, \omega=0$ is written in the form;

$$
\left\{\mathcal{G}^{+}(0)\right\}^{-1}=\left[\begin{array}{ccccc}
0 & \widetilde{t}_{12}^{C} & 0 & \widetilde{t}_{14}^{C} & \cdots \\
\widetilde{t}_{21}^{C} & 0 & \widetilde{t}_{23}^{C} & 0 & \cdots \\
0 & \widetilde{t}_{32}^{C} & 0 & \widetilde{t}_{34}^{C} & \cdots \\
\widetilde{t}_{41}^{C} & 0 & \widetilde{t}_{43}^{C} & 0 & \cdots \\
\vdots & \vdots & \vdots & \vdots & \ddots
\end{array}\right]+\left[\begin{array}{ccc}
i \Gamma_{L}(0) & & \\
& 0 & \\
& & i \Gamma_{R}(0)
\end{array}\right],
$$

and $G_{i j}^{+}(0)$ is obtained by taking the inverse of Eq. (23). Specifically, using the explicit form of $G_{1 N}^{+}(0)$, the de conductance $g_{N}$ can be obtained from Eq. (14). For $N=1$ and 2 , the dc conductance is written in the form

$$
\begin{aligned}
& g_{1}=\frac{2 e^{2}}{h} \frac{\Gamma_{L} \Gamma_{R}}{\left[\left(\Gamma_{L}+\Gamma_{R}\right) / 2\right]^{2}}, \\
& g_{2}=\frac{2 e^{2}}{h} \frac{\Gamma_{L} \Gamma_{R}\left\{\widetilde{t}_{12}^{C}\right\}^{2}}{\left[\left(\Gamma_{L} \Gamma_{R}+\left\{\widetilde{t}_{12}^{C}\right\}^{2}\right) / 2\right]^{2}} .
\end{aligned}
$$

Here $\Gamma_{L} \equiv \Gamma_{L}(0), \Gamma_{R} \equiv \Gamma_{R}(0)$, and we will use this simplified notation in what follows. For $N \geq 3, G_{1 N}^{+}(0)$ is written in the form

$$
\begin{aligned}
& G_{1 N}^{+}(0)=(-1)^{N+1} \frac{\operatorname{det} \mathcal{K}_{N 1}}{\operatorname{det}\left\{\mathcal{G}^{+}(0)\right\}^{-1}}, \\
& \operatorname{det}\left\{\mathcal{G}^{+}(0)\right\}^{-1}=\left\{\begin{array}{ll}
i \Gamma_{L} \operatorname{det} \mathcal{K}_{11}+i \Gamma_{R} \operatorname{det} \mathcal{K}_{N N}, & \text { for odd } N \\
-\Gamma_{L} \Gamma_{R} \operatorname{det} \mathcal{K}_{11}^{N N}+\operatorname{det} \mathcal{K}, & \text { for even } N
\end{array} .\right.
\end{aligned}
$$

Here $\mathcal{K}_{i j}$ is a $(N-1) \times(N-1)$ matrix obtained from $\mathcal{K}$ by deleting the $i$-th row and the $j$-th column. Similarly, $\mathcal{K}_{11}^{N N}$ is a $(N-2) \times(N-2)$ matrix obtained from $\mathcal{K}$ by deleting the first and the $N$-th rows, and the first and the $N$-th columns. Due to the checkered structure of Eq. (23), the determinants satisfy a relation

$$
\left(\operatorname{det} \mathcal{K}_{N 1}\right)^{2}= \begin{cases}\operatorname{det} \mathcal{K}_{11} \operatorname{det} \mathcal{K}_{N N}, & \text { for } \text { odd } N \\ -\operatorname{det} \mathcal{K} \operatorname{det} \mathcal{K}_{11}^{N N}, & \text { for } \text { even } N\end{cases}
$$


Although the details are given in the appendix $\mathbb{A}$, the origin of the even-odd dependence can be understood just seeing typical examples;

$$
\begin{aligned}
\mathcal{K} & =\left[\begin{array}{ccccc}
0 & \widetilde{t}_{12}^{C} & 0 & \widetilde{t}_{41}^{C} & 0 \\
\widetilde{t}_{12}^{C} & 0 & \widetilde{t}_{23}^{C} & 0 & \widetilde{t}_{52}^{C} \\
0 & \widetilde{t}_{23}^{C} & 0 & \widetilde{t}_{34}^{C} & 0 \\
\widetilde{t}_{41}^{C} & 0 & \widetilde{t}_{34}^{C} & 0 & \widetilde{t}_{45}^{C} \\
0 & \widetilde{t}_{52}^{C} & 0 & \widetilde{t}_{45}^{C} & 0
\end{array}\right] \quad \text { for } N=5 \\
\mathcal{K} & =\left[\begin{array}{cccccc}
0 & \widetilde{t}_{12}^{C} & 0 & \widetilde{t}_{41}^{C} & 0 & \widetilde{t}_{61}^{C} \\
\widetilde{t}_{12}^{C} & 0 & \widetilde{t}_{23}^{C} & 0 & \widetilde{t}_{52}^{C} & 0 \\
0 & \widetilde{t}_{23}^{C} & 0 & \widetilde{t}_{34}^{C} & 0 & \widetilde{t}_{63}^{C} \\
\widetilde{t}_{41}^{C} & 0 & \widetilde{t}_{34}^{C} & 0 & \widetilde{t}_{45}^{C} & 0 \\
0 & \widetilde{t}_{52}^{C} & 0 & \widetilde{t}_{45}^{C} & 0 & \widetilde{t}_{56}^{C} \\
\widetilde{t}_{61}^{C} & 0 & \widetilde{t}_{63}^{C} & 0 & \widetilde{t}_{56}^{C} & 0
\end{array}\right] \quad \text { for } N=6 .
\end{aligned}
$$

The antidiagonal elements, $\mathcal{K}_{j, N+1-j}$ for $j=1,2, \ldots, N$, are zero for odd $N$, while those are not for even $N$. Furthermore, $\operatorname{det} \mathcal{K}$ is zero for odd $N$, while it is finite and can be divided into two determinants for even $N$. Using the properties Eqs. (26)-(28) with Eq. (14), the dc conductance for odd $N(=2 M+1)$ is written in the form

$$
g_{2 M+1}=\frac{2 e^{2}}{h} \frac{\widetilde{\Gamma}_{L} \widetilde{\Gamma}_{R}}{\left[\left(\widetilde{\Gamma}_{L}+\widetilde{\Gamma}_{R}\right) / 2\right]^{2}},
$$

where $\widetilde{\Gamma}_{L}=\lambda \Gamma_{L}, \widetilde{\Gamma}_{R}=\Gamma_{R} / \lambda$, and

$$
\lambda=\sqrt{\frac{\operatorname{det} \mathcal{K}_{11}}{\operatorname{det} \mathcal{K}_{N N}}} .
$$

We note that the parameter $\lambda$ can be simplified as Eq. (A10) using the checkered structure further. If the system also has the inversion symmetry $\Gamma_{L}=\Gamma_{R}$ in addition to the electronhole symmetry, the parameter is fixed as $\lambda=1$. This is because the matrix $\mathcal{K}$ becomes symmetric with respect to the antidiagonal line. Therefore, due to the combination of the symmetries, the perfect transmission occurs $g_{2 M+1}=2 e^{2} / h$ independent of $M$ and the details 
of the interaction, as far as the perturbation expansion is valid. Physically, this is caused by the Kondo resonance appearing at the Fermi energy $\omega=0$. On the other hand, for even $N(=2 M)$, the dc conductance is written in the form

$$
\begin{aligned}
g_{2 M} & =\frac{2 e^{2}}{h} \frac{\Gamma_{L} \Gamma_{R} \widetilde{v}_{C}^{2}}{\left[\left(\Gamma_{L} \Gamma_{R}+\widetilde{v}_{C}^{2}\right) / 2\right]^{2}} \\
\widetilde{v}_{C}^{2} & =-\frac{\operatorname{det} \mathcal{K}}{\operatorname{det} \mathcal{K}_{11}^{N N}} .
\end{aligned}
$$

We note that the parameter $\widetilde{v}_{C}$ can also be simplified as Eq. (A6). These two expressions for the dc conductance, Eqs. (31) and (33), are the main results of this section.

We next examine the Friedel sum rule. Using the property Eq. (27) and the expression of the total charge displacement Eqs. (16) and (17), we obtain $\operatorname{det} \boldsymbol{S}=1$ for even $N$, and $\operatorname{det} \boldsymbol{S}=-1$ for odd $N$. This is consistent with the fact that the average number of electrons in each site is unity. In order to realize the electron-hole symmetry, the bare matrix element $t_{i j}^{C}$ should also have the checkered structure as that is assumed for the renormalized parameter $\widetilde{t}_{i j}^{C}$, i.e., the system must be classified into two sublattices. Note that, since only the symmetry is assumed so far, the bare hopping matrix element is not necessary to be restricted to the nearest-neighbor one. Furthermore, the system is possible to be disordered through the randomness in the off-diagonal element of $t_{i j}^{C}$.

\section{TRANSPORT THROUGH A FINITE HUBBARD CHAIN}

In this section, we will use the effective Hamiltonian to investigate the conductance of a finite Hubbard chain connected to two semi-infinite leads. The system is considered as a model for a series of quantum dots or atomic wire of nanometer size. We calculate the renormalized hopping matrix element $\widetilde{t}_{i j}^{C}$ up to the second order in $U$, and obtain the dc conductance using the expressions given in the previous section. We note that the secondorder perturbation theory has been used by several groups for studying transport properties of a single impurity 514,43 and systems consisting of a number of resonant levels.4.45 For 
$N \geq 2$, the off-diagonal part of the self-energy plays an important role on the conductance at $T=0$ as seeing in the results presented below. To our knowledge, however, effects of the off-diagonal part have not been examined sufficiently so far.

The schematic picture of the model is illustrated in Fig. 2: the system consists of $N$ interacting sites at the center $1 \leq i \leq N$, and two non-interacting leads at $-\infty<i \leq 0$ and $N+1 \leq i<+\infty$. The explicit form of the Hamiltonian is given by

$$
\begin{aligned}
H= & H_{0}+H_{I}, \\
H_{0}= & -\sum_{i=-\infty}^{+\infty} \sum_{\sigma} t_{i}\left(c_{i+1 \sigma}^{\dagger} c_{i \sigma}+c_{i \sigma}^{\dagger} c_{i+1 \sigma}\right)-\mu \sum_{i=-\infty}^{+\infty} \sum_{\sigma} c_{i \sigma}^{\dagger} c_{i \sigma} \\
& +\sum_{i=1}^{N} \sum_{\sigma}\left(\epsilon_{0}+\frac{U}{2}\right) n_{i \sigma}, \\
H_{I}= & U \sum_{i=1}^{N}\left[n_{i \uparrow} n_{i \downarrow}-\frac{1}{2}\left(n_{i \uparrow}+n_{i \downarrow}\right)\right],
\end{aligned}
$$

where $U$ is the repulsive interaction in the chain, $\epsilon_{0}$ is the on-site energy, and $n_{i \sigma}=c_{i \sigma}^{\dagger} c_{i \sigma}$. We take the hopping matrix element to be $t_{i}=t$ for every links except for the coupling between the chain and leads, i.e., $t_{0}=v_{L}$ and $t_{N}=v_{R}$. Thus, the level width caused by the coupling with the leads is given by $\Gamma_{\alpha}(0)=\pi v_{\alpha}^{2} D(0)$ for $\alpha=L, R$ with $D(0)=\sqrt{4 t^{2}-\mu^{2}} /\left(2 \pi t^{2}\right)$. In what follows, we concentrate on the electron-hole symmetric case taking the parameters to be $\mu=0$ and $\epsilon_{0}=-U / 2$.

Within the second order, the self-energy correction is described by a diagram Fig. 3, and the value at $T=0, \omega=0$ is obtained from the expression

$$
\Sigma_{i j}^{(2)}\left(i 0^{+}\right)=-U^{2} \int_{-\infty}^{\infty} \int_{-\infty}^{\infty} \frac{d \epsilon d \epsilon^{\prime}}{(2 \pi)^{2}} G_{i j}^{(0)}(i \epsilon) G_{i j}^{(0)}\left(i \epsilon^{\prime}\right) G_{j i}^{(0)}\left(i \epsilon+i \epsilon^{\prime}\right)
$$

for $1 \leq i, j \leq N$. Here $G_{i j}^{(0)}(i \epsilon)$ is the unperturbed Green's function corresponding to $H_{0}$, and thus $\Sigma_{i j}^{(2)}\left(i 0^{+}\right)$depends on $v_{L}$ and $v_{R}$ through $G_{i j}^{(0)}$. Note that the value of the retarded function at $\omega=0$ can be obtained from the Matsubara function, i.e., $\Sigma_{i j}^{(2)}\left(i 0^{+}\right)=$ $\left.\Sigma_{i j}^{(2)}(i \epsilon)\right|_{\epsilon \rightarrow 0^{+}}$. The imaginary part of the self-energy vanishes owing to the Fermi-liquid property, i.e., $\operatorname{Im} \Sigma_{i j}^{(2)}\left(i 0^{+}\right)=0$. Furthermore, $\Sigma_{i j}^{(2)}\left(i 0^{+}\right)=0$ for even $|i-j|$ since $G_{i j}^{(0)}(-i \epsilon)=$ 
$(-1)^{|i-j|+1} G_{i j}^{(0)}(i \epsilon)$ in the electron-hole symmetric case. We have done the integration Eq. (38) numerically to estimate the renormalized matrix element $\widetilde{t}_{i j}^{C}$ defined by Eq. (13) within the second order, and obtain the dc conductance $g_{N}$ using Eqs. (31) and (33). As an example, we show the result of the self-energy for $N=6$,

$$
\begin{aligned}
\boldsymbol{\Sigma}^{(2)}\left(i 0^{+}\right) & =-t\left(\frac{U}{2 \pi t}\right)^{2} \widetilde{\boldsymbol{\Sigma}}^{(2)}, \\
\widetilde{\boldsymbol{\Sigma}}^{(2)} & =\left[\begin{array}{cccccc}
0 & 0.8596 & 0 & -0.1642 & 0 & 0.0783 \\
0.8596 & 0 & 0.3969 & 0 & -0.0352 & 0 \\
0 & 0.3969 & 0 & 0.7543 & 0 & -0.1642 \\
-0.1642 & 0 & 0.7543 & 0 & 0.3969 & 0 \\
0 & -0.0352 & 0 & 0.3969 & 0 & 0.8596 \\
0.0783 & 0 & -0.1642 & 0 & 0.8596 & 0
\end{array}\right] .
\end{aligned}
$$

Here the coupling is taken to be $v_{L} / t=v_{R} / t=0.8$. The matrix element for a given distance $|i-j|$ shows an oscillatory behavior, i.e., $\Sigma_{l, l+2 m+1}^{(2)}\left(i 0^{+}\right)$is an oscillatory function of $l$. In contrast, in the special case $v_{L}=v_{R}=t, \Sigma_{l, l+2 m+1}^{(2)}\left(i 0^{+}\right)$is independent of $l$ and the integration Eq. (38) can be done analytically. 30 Note that $\Sigma_{i j}^{(2)}\left(i 0^{+}\right)$is also an oscillatory function of $|i-j|$, and the absolute value tends to decrease with increasing $|i-j|$.

\section{A. Symmetric connection $v_{L}=v_{R}$}

We now examine the case where the system has the inversion symmetry, $v_{L}=v_{R} \equiv v$, in addition to the electron-hole symmetry. In this case, as it was shown generally in the previous section, the perfect transmission occurs for odd $N$ independent of $U$. Physically, this is due to the contribution of the Kondo resonance appearing at the Fermi energy. So, we have done the numerical calculation only for even $N$. The result of $g_{N}$ is plotted as a function of the size $N$ in Fig. 1 , where the parameters are taken to be $v / t=0.8$ and $U /(2 \pi t)=0.4$. The result shows a typical even-odd oscillatory behavior, and the conductance for even $N$ decreases with increasing $N$. This reduction is caused by the oscillatory $l$ dependence of 
the self-energy $\Sigma_{l, l+2 m+1}^{(2)}$. In Fig. 5, the envelope of $g_{N}$ for even $N(=2 M)$ is plotted vs $M$ for several values of the repulsion $U /(2 \pi t)=0.0,0.2,0.4,0.6$, and 0.8 , taking the mixing matrix element to be $v / t=0.8$. The conductance $g_{2 M}$ decreases with increasing $M$ and increasing $U$. In the non-interacting case, $g_{2 M}$ is independent of $M$ since the parameter defined by Eq. (34) is simply $\widetilde{v}_{C}=t$. The reduction of the conductance seems to show a tendency toward a Mott-Hubbard insulator. However, the result shows a rather moderate decay, i.e., it does not show an exponential dependence. Since the range of $U$ in which the second-order perturbation theory is able to provide quantitatively reliable results depends on the size $M$, contributions of higher-order terms should be examined in order to clarify the correct asymptotic behavior for large $M$.

In Fig. 6, the conductance is plotted as a function of $U$ for a number of even $N(=$ $2,4,6, \ldots)$ taking the parameters to be $v / t=0.8$ (dashed lines) and $v / t=1.0$ (solid lines). As it can be seen in the behavior of the dashed lines, the reduction of $g_{2 M}$ is proportional to $U^{2}$ for small $U$ when $|v / t|<1.0$. The curvature increases with the size $M$. In order to see this quantitatively, we expand the conductance in powers of $U$ as $g_{2 M}=\left(2 e^{2} / h\right)\left[C_{0}-\right.$ $\left.C_{2}\{U /(2 \pi t)\}^{2}+\cdots\right]$, and plot the ratio $C_{2} / C_{0}$ as a function of $M$ in Fig. 7 for several values of $v / t(=0.1,0.2, \ldots, 0.9) . C_{2}$ increases with the size $M$, and seems to diverge in the limit of large $M$ showing a power low behavior. Note that the transmission probability in the non-interacting case $C_{0}$ depends on the mixing matrix element $v$ but is independent of the size $M$ as mentioned in the above. Furthermore, since the self-energy is calculated up to the order $U^{2}$, the result of $C_{2}$ is exact. The ratio $C_{2} / C_{0}$ increases with decreasing $v$. This means that the effect of the interaction is enhanced when the coupling between the sample and leads is weak. In the special case $v=t$, the reduction of $g_{2 M}$ is proportional to $U^{4}$ for small $U$, i.e., $g_{2 M}=\left(2 e^{2} / h\right)\left[1-C_{4}\{U /(2 \pi t)\}^{4}+\cdots\right]$. This is because the unperturbed system described by $H_{0}$ has a translational invariance accidentally in this case and the reflection probability is zero at $U=0$. The solid lines for $N \gtrsim 10$ in Fig. 6 are almost overlapping each other. In Fig. 8, $C_{4}$ is plotted vs $M$. This result is also exact, and the coefficient $C_{4}$ 
converges to a finite value for large $M ; C_{4} \simeq 0.5293$ for $N=250$. In the appendix $\mathbb{B}$, the convergence of $C_{4}$ in the limit of $M \rightarrow \infty$ is confirmed with another approach. Therefore, when $v=t, g_{2 M}$ is finite even in the limit of large $M$ at least for small $U .30$ For large values of $U$, both the dashed and solid lines in Fig. 6 tends to zero showing a $1 / U^{4}$ dependence. However this behavior depends on the approximation: as it can be confirmed from Eqs. (33) and (34), $\widetilde{v}_{C} \propto U^{n}$ and $g_{2 M} \propto 1 / U^{2 n}$ for large $U$ when the self-energy is estimated within the $n$-th order perturbation in $U$.

The mixing matrix element $v$ determines the bare level width of the resonant states, and in the present case it is given by $\Gamma=v^{2} / t$. Since we are now considering the electronhole symmetric case, the Fermi level for even $N$ is located between the two resonant states corresponding to the highest occupied and lowest unoccupied levels. In Fig. 9, $g_{2 M}$ is plotted vs $U$ for several values of $v / t(=0.4,0.5,0.6, \ldots, 1.0)$, where the solid (dashed) lines are the results for $N=40(N=4)$. The value of the conductance itself decreases with $v$, but the qualitative feature of the $U$ dependence is similar in the cases of $|v / t|<1.0$. In Fig. [10, we show the conductance as a function of $M$ for several $v / t(=0.4,0.5, \ldots, 1.0)$ taking the repulsion to be $U /(2 \pi t)=0.3$. In this figure, the conductance is normalized by the

non-interacting value $g_{2 M}^{(0)} \equiv\left(2 e^{2} / h\right) C_{0}$ which depends on $v$. The normalized conductance decreases with $v$. This also means that the effect of the interaction is enhanced when the level width of the resonant states is small.

\section{B. Asymmetric connection $v_{L} \neq v_{R}$}

We next examine the case where the inversion symmetry is broken, $v_{L} \neq v_{R}$, but the electron-hole symmetry is still preserved in the equilibrium state by the condition $\mu=0$ and $\epsilon_{0}=-U / 2$. As one typical example, we take the mixing parameter to be $v_{L} / t=0.8$ and $v_{R} / t=0.6$ in this subsection. In Fig. 11 (a) and (b), the conductance is plotted as a function of the size $N$ taking $U /(2 \pi t)$ to be (a) 0.2 and (b) 0.4 , respectively. The result shows the even-odd oscillatory behavior as that in the case of $v_{L}=v_{R}$ discussed in the 
above. However, in the case of $v_{L} \neq v_{R}$, the perfect transmission does not occur as it can be deduced from Eq. (31), so that we have done the numerical calculation also for odd $N$. Although the Kondo resonance is still present at the Fermi level owing to the electronhole symmetry, the conductance is reduced. Nevertheless, the Kondo state contributes to the higher transmission for odd $N$. In Fig. 12, $g_{N}$ is plotted as a function of $U$ for a number of $N(=1,2,3, \ldots, 40)$. For even $N$, qualitative features of the results are similar to those in the inversion symmetric case [See Fig. 6]. On the other hand, for odd $N$, the features of the curves are somewhat different. However, the results for large values of $U$ have some limitations, because the parameter $\lambda$ defined by Eq. (32) tends to an incorrect limit for large $U$ when the self-energy is estimated within a finite order in $U$, i.e., $\lambda$ tends to the ratio of the highest-order term of the numerator and that of the denominator of Eq. (A10). In the non-interacting case the conductance for even and odd $N, g_{2 M}$ and $g_{2 M+1}$, are independent of the size $M$, respectively, since $\widetilde{v}_{C}=t$ and $\lambda=1$. For small $U$, the reduction of the conductance is proportional to $U^{2}$ and the curvature increases with the size $M$, except for the single impurity case $N=1$. In Fig. 13, the coefficient $C_{2}$ defined by $g_{N}=\left(2 e^{2} / h\right)\left[C_{0}-C_{2}\{U /(2 \pi t)\}^{2}+\cdots\right]$ is plotted as a function of $N$. In the figure, the envelop for even $N$ is in upward from that for odd $N$. The coefficient $C_{2}$ increases with the size $N$ showing an oscillatory behavior, and probably it diverges in the limit of large $N$.

\section{SUMMARY}

We have applied a quasi-particle description of a Fermi liquid to the transport through a small interacting system connected to reservoirs. With this approach, we have studied the properties of quasi-particles in an electron-hole symmetric case. In this case, effects of electron correlation come in the theory through the off-diagonal part of the self-energy, and the conductance can be written in a simplified form Eqs. (31) or (33) depending on whether the number of the interacting sites $N$ is even or odd. It is shown using Eq. (31) that the perfect transmission occurs quite generally for odd $N$ if the system has an inversion 
symmetry in addition to the electron-hole symmetry. We have applied the method to a small Hubbard chain of finite size $N$ with the second-order perturbation theory in $U$, and examine the conductance as a function of $N, U$, and the coupling between the chain and the leads, i.e., $v_{L}$ and $v_{R}$. Effects of electron correlation are enhanced in weak coupling case $v_{L}, v_{R}<t$. In this case, the conductance for even $N$ tends to zero for large $N$. This behavior is qualitatively different from that in the special case $v_{L}=v_{R}=t$. This seems to be caused by the difference in the structure of the resonant states. Quantitatively, the range of $U$ in which the second-order perturbation theory is able to provide reliable results tends to be narrow with increasing $N$, and thus the higher order terms should be included in order to refine the results obtained for large $U$ and to clarify the asymptotic behavior for large $N$.

Throughout this work, we have assumed that the interaction is switched on only in the sample region at the center. When the interaction is switched on also in the two leads, the description in terms of the quasi-particles is still possible if the ground state is changed continuously against this interaction. But some modifications are necessary. The interaction in the leads will cause the renormalization of the incoming and outgoing fields corresponding to initial and final states of the scattering matrix, i.e., the renormalization of the external

lines. Furthermore, the quasi-particle description can be extended to finite temperatures by taking into account the residual interaction among the quasi-particles or the contributions of vertex corrections. Especially, an overall picture of the even-odd property described in the last part of Sec. 【1 will be confirmed microscopically based on the finite temperature theory.

\section{ACKNOWLEDGMENTS}

We would like to thank H. Fukuyama, H. Ishii, W. Izumida, N. Nagaosa, S. Nonoyama, and O. Sakai for valuable discussions. This work is partially supported by the Grand-in-Aid for Scientific Research from the Ministry of Education, Science and Culture, Japan. 


\section{APPENDIX A: $\mathcal{K}$ MATRIX IN THE ELECTRON-HOLE SYMMETRIC CASE}

We summarize here properties of the renormalized hopping matrix $\mathcal{K}$, and provide the derivation of Eq. (28). In the electron-hole symmetric case, $\mathcal{K}$ has a checkered structure as shown in Eqs. (29) and (30). Thus, $\operatorname{det} \mathcal{K}=0$ for odd $N$. For even $N(=2 M)$, the determinant can be divided into two parts;

$$
\left|\begin{array}{ccccc}
0 & \widetilde{t}_{12}^{C} & 0 & \widetilde{t}_{14}^{C} & \cdots \\
\widetilde{t}_{21}^{C} & 0 & \widetilde{t}_{23}^{C} & 0 & \cdots \\
0 & \widetilde{t}_{32}^{C} & 0 & \widetilde{t}_{34}^{C} & \cdots \\
\widetilde{t}_{41}^{C} & 0 & \widetilde{t}_{43}^{C} & 0 & \cdots \\
\vdots & \vdots & \vdots & \vdots & \ddots
\end{array}\right|=(-1)^{M} \operatorname{det} \boldsymbol{Q} \operatorname{det} \boldsymbol{Q}^{\prime} .
$$

Here $\boldsymbol{Q}$ and $\boldsymbol{Q}^{\prime}$ are $M \times M$ matrices defined by

$$
\boldsymbol{Q}=\left[\begin{array}{ccc}
\widetilde{t}_{12}^{C} & \widetilde{t}_{14}^{C} & \cdots \\
\widetilde{t}_{32}^{C} & \widetilde{t}_{34}^{C} & \cdots \\
\vdots & \vdots & \ddots
\end{array}\right], \quad \boldsymbol{Q}^{\prime}=\left[\begin{array}{ccc}
\widetilde{t}_{21}^{C} & \widetilde{t}_{23}^{C} & \cdots \\
\widetilde{t}_{41}^{C} & \widetilde{t}_{43}^{C} & \cdots \\
\vdots & \vdots & \ddots
\end{array}\right]
$$

Since the renormalized parameter is real and $\widetilde{t}_{i j}^{C}=\widetilde{t}_{j i}^{C}$ owing to the time-reversal symmetry, $\operatorname{det} \boldsymbol{Q}=\operatorname{det} \boldsymbol{Q}^{\prime}$. Consequently, for even $N(=2 M)$, the determinant of the matrices $\mathcal{K}$, $\mathcal{K}_{11}^{N N}$, and $\mathcal{K}_{N 1}$ can be factorized as

$$
\begin{aligned}
\operatorname{det} \mathcal{K} & =(-1)^{M}\{\operatorname{det} \boldsymbol{Q}\}^{2}, \\
\operatorname{det} \mathcal{K}_{11}^{N N} & =(-1)^{M-1}\left\{\operatorname{det} \boldsymbol{Q}_{11}^{N N}\right\}^{2}, \\
\operatorname{det} \mathcal{K}_{N 1} & =\operatorname{det} \boldsymbol{Q} \operatorname{det} \boldsymbol{Q}_{11}^{N N} .
\end{aligned}
$$

Here $\boldsymbol{Q}_{11}^{N N}$ is a $(M-1) \times(M-1)$ matrix extracted from $\mathcal{K}_{11}^{N N}$ in the similar way as it was done for extracting $\boldsymbol{Q}$ from $\mathcal{K}$. Using Eqs. (A3)-(A5), we obtain Eq. (28) for even $N$, i.e., $\left(\operatorname{det} \mathcal{K}_{N 1}\right)^{2}=-\operatorname{det} \mathcal{K} \operatorname{det} \mathcal{K}_{11}^{N N}$. Furthermore, $\widetilde{v}_{C}$ defined by Eq. (34) is simplified as

$$
\widetilde{v}_{C}=\left|\frac{\operatorname{det} \boldsymbol{Q}}{\operatorname{det} \boldsymbol{Q}_{11}^{N N}}\right| .
$$

For odd $N(=2 M+1)$, the $2 M \times 2 M$ matrices $\mathcal{K}_{11}$ and $\mathcal{K}_{N N}$ have the similar properties described in the above. Consequently, the determinants can be factorized as 


$$
\begin{aligned}
\operatorname{det} \mathcal{K}_{11} & =(-1)^{M}\left\{\operatorname{det} \boldsymbol{Q}_{11}\right\}^{2}, \\
\operatorname{det} \mathcal{K}_{N N} & =(-1)^{M}\left\{\operatorname{det} \boldsymbol{Q}_{N N}\right\}^{2}, \\
\operatorname{det} \mathcal{K}_{N 1} & =\operatorname{det} \boldsymbol{Q}_{11} \operatorname{det} \boldsymbol{Q}_{N N} .
\end{aligned}
$$

Here $\boldsymbol{Q}_{11}$ and $\boldsymbol{Q}_{N N}$ are $M \times M$ matrices extracted from $\mathcal{K}_{11}$ and $\mathcal{K}_{N N}$, respectively, as it was done in the above. Using Eq. (A7)-(A9), we obtain Eq. (28) for odd $N$, i.e., $\left(\operatorname{det} \mathcal{K}_{N 1}\right)^{2}=$ $\operatorname{det} \mathcal{K}_{11} \operatorname{det} \mathcal{K}_{N N}$. Also, $\lambda$ defined by Eq. (32) is simplified as

$$
\lambda=\left|\frac{\operatorname{det} \boldsymbol{Q}_{11}}{\operatorname{det} \boldsymbol{Q}_{N N}}\right| .
$$

\section{APPENDIX B: REFLECTION COEFFICIENT IN A SPECIAL CASE}

We show here another approach to clarify the asymptotic behavior of the coefficient $C_{4}$ introduced in Sec. प in the case of $v_{L}=v_{R}=t$. In this case, the Dyson equation Eq. (9) is written in terms of the scattering matrix $\mathcal{T}_{l l^{\prime}}$ as,

$$
G_{j j^{\prime}}=G_{j j^{\prime}}^{(0)}+\sum_{l, l^{\prime}=1}^{N} G_{j l}^{(0)} \mathcal{T}_{l l^{\prime}} G_{l^{\prime} j^{\prime}}^{(0)} .
$$

The transmission and reflection coefficients are also written in terms of $\mathcal{T}_{l l^{\prime}}$ as $\widetilde{t}(0)=1-$ $i \mathcal{T}_{k_{F} k_{F}}\left(i 0^{+}\right) / v_{F}$ and $\widetilde{r}(0)=-i \mathcal{T}_{-k_{F} k_{F}}\left(i 0^{+}\right) / v_{F}, 1,199$ where $v_{F}=2 t, k_{F}=\pi / 2$, and

$$
\mathcal{T}_{k k^{\prime}}=\sum_{l, l^{\prime}=1}^{N} e^{-i k l} \mathcal{T}_{l l^{\prime}} e^{i k^{\prime} l^{\prime}} .
$$

Since the unperturbed system has the translational invariance in the present case, the lowestorder scattering matrix is given by $\mathcal{T}_{l l^{\prime}}^{(2)}=\Sigma_{l l^{\prime}}^{(2)}$. Thus, using a wavenumber representation of $\Sigma_{l l^{\prime}}^{(2)}, 30$ the lattice sum Eq. (B2) can be done explicitly, and the reflection coefficient is expressed as

$$
\begin{aligned}
\widetilde{r}_{N}^{(2)}= & -\frac{i}{v_{F}} e^{i k_{F}(N+1)} U^{2} \int \frac{d k_{3} d k_{2} d k_{1}}{(2 \pi)^{3}} \frac{\cos k_{F} N-\cos \left[\left(k_{1}+k_{2}-k_{3}\right) N\right]}{\cos k_{F}-\cos \left(k_{1}+k_{2}-k_{3}\right)} \\
& \times \mathrm{P} \frac{f_{k_{3}}\left(1-f_{k_{2}}\right)\left(1-f_{k_{1}}\right)+\left(1-f_{k_{3}}\right) f_{k_{2}} f_{k_{1}}}{\xi_{k_{3}}-\xi_{k_{2}}-\xi_{k_{1}}} .
\end{aligned}
$$


Here $\xi_{k}=-2 t \cos k, f_{k}=\left[e^{\beta \xi_{k}}+1\right]^{-1}$, and $\mathrm{P}$ denotes the Cauchy principal value. It can be confirmed from Eq. (B3) that $\widetilde{r}_{N}^{(2)}=0$ for odd $N$. For large even $N$, the contribution of the fast varying $\cos \left[\left(k_{1}+k_{2}-k_{3}\right) N\right]$ part becomes small, and the dominant contribution comes form the $\cos k_{F} N$ part. Thus, for large even $N(=2 M)$, the reflection coefficient is written in the form $\lim _{M \rightarrow \infty} \widetilde{r}_{2 M}^{(2)}=\sqrt{C_{4}^{\infty}}\{U /(2 \pi t)\}^{2}$ with

$$
\sqrt{C_{4}^{\infty}} \equiv \frac{1}{4 \pi} \mathrm{P} \int_{-\pi / 2}^{\pi / 2} \frac{d k_{3} d k_{2} d k_{1}}{\left[\cos k_{1}+\cos k_{2}+\cos k_{3}\right] \cos \left(k_{1}+k_{2}+k_{3}\right)} .
$$

The coefficient $\sqrt{C_{4}^{\infty}}$ is finite and estimated numerically as $\sqrt{C_{4}^{\infty}} \simeq 0.729$. Thus, in the limit of large even $N$, the dc conductance in the case of $v_{L}=v_{R}=t$ is written in the form $\lim _{M \rightarrow \infty} g_{2 M}=\left(2 e^{2} / h\right)\left[1-C_{4}^{\infty}\{U /(2 \pi t)\}^{4}+\cdots\right]$. 


\section{REFERENCES}

${ }^{1}$ T. K. Ng and P. A. Lee, Phys. Rev. Lett. 61, 1768 (1988).

${ }^{2}$ L. I. Glazman and M. E. Raikh, Pis'ma Zh. Eksp. Teor. Fiz. 47, 378 (1988) [JETP Lett. 47, $452(1988)]$.

${ }^{3}$ A. Kawabata, J. Phys. Soc. Jpn. 60, 3222 (1991).

${ }^{4}$ Y. Meir, N. S. Wingreen, and P. A. Lee, Phys. Rev. Lett. 66, 3048 (1991); Phys. Rev. Lett. 70, 2601 (1993).

${ }^{5}$ S. Hersfield, J. H. Davies, and J. W. Wilkins, Phys. Rev. B 46, 7046 (1992).

${ }^{6}$ D. C. Ralph and R. A. Buhrman, Phys. Rev. Lett. 72, 3401 (1994).

${ }^{7}$ D. Goldharber-Gordon, H. Shtrikman, D. Mahalu, D. Abusch-Magder, U. Meirav, and M. A. Kastner, Nature 391, 156 (1998).

${ }^{8}$ S. M. Cronenwett, T. H. Oosterkamp, and L. P. Kouwenhoven, Science 281, 540 (1998).

${ }^{9}$ C. L. Kane and M. P. A. Fisher, Phys. Rev. Lett. 68, 1220 (1992).

${ }^{10}$ A. Furusaki and N. Nagaosa, Phys. Rev. B 474631 (1993).

${ }^{11}$ S. Tarucha, T. Honda, and T. Saku, Solid State Commun. 94 (1995) 413.

${ }^{12}$ Y. Meir and N. S. Wingreen, Phys. Rev. Lett. 68, 2512 (1992).

${ }^{13}$ L. V. Keldysh, Zh. Eksp. Teor. Fiz. 47, 1515 (1964) [Sov. Phys. JETP 20, 1018 (1965)].

${ }^{14}$ C. Caroli, R. Combescot, P. Nozières, and D. Saint-James, J. Phys. C 4, 916 (1971).

${ }^{15}$ E. N. Economou and C. M. Soukoulis: Phys. Rev. Lett 46 (1981) 618.

${ }^{16}$ D. S. Fisher and P. A. Lee, Phys. Rev. B 23, 6851 (1981).

${ }^{17}$ P. A. Lee and D. S. Fisher, Phys. Rev. Lett. 47, 882 (1981).

${ }^{18}$ A. Oguri, J. Phys. Soc. Jpn. 66, 1427 (1997). 
${ }^{19}$ A. Oguri, Phys. Rev. B 56, 13422 (1997); 58, 1690(E) (1998) .

${ }^{20}$ A. C. Hewson, Adv. Phys. 43, 543 (1994); The Kondo Problem to Heavy Fermions (Cambridge University Press, Cambridge, 1993).

${ }^{21}$ W. Izumida, O. Sakai, and Y. Shimizu, J. Phys. Soc. Jpn. 66, 717 (1997); J. Phys. Soc. Jpn. 67, 2444 (1998).

${ }^{22}$ O. Sakai, S. Suzuki, W. Izumida, and A. Oguri, J. Phys. Soc. Jpn. 681640 (1999).

${ }^{23}$ A. Oguri, H. Ishii, and T. Saso, Phys. Rev. B 51, 4715 (1995).

${ }^{24}$ V. V. Ponomarenko and N. Nagaosa Phys. Rev. Lett. 81, 2304 (1998).

${ }^{25}$ A. A. Odintsov, Y. Tokura and S. Tarucha, Phys. Rev. B 56, R12729 (1997).

${ }^{26}$ O. A. Starykh and D. L. Maslov, Phys. Rev. Lett. 80, 1694 (1998).

${ }^{27}$ M. Mori, M. Ogata and H. Fukuyama, J. Phys. Soc. Jpn. 66, 3363 (1997).

${ }^{28}$ S. Fujimoto and N. Kawakami, J. Phys. Soc. Jpn. 65, 3700 (1996).

${ }^{29}$ K. Yosida and K. Yamada, Prog. Theor. Phys. Suppl. No. 46, 244 (1970); K. Yamada, Prog. Theor. Phys. 53, 970 (1975).

${ }^{30}$ A. Oguri, Phys. Rev. B 59, 12240 (1999).

${ }^{31}$ A. Oguri, Physica B 284-288, 1932 (2000).

${ }^{32}$ V. Zlatić and V. Horvatić, Phys. Rev. B 28, 6940 (1983).

${ }^{33}$ J. S. Langer and V. Ambegaokar, Phys. Rev. 121, 1090 (1961).

${ }^{34}$ We note that Eq. (14) has been derived for noninteracting systems by Caroli et al 14 Also, Pernas et al have obtained the similar expression for interacting systems with the single-site approximation neglecting the off-diagonal part of the self-energy.

${ }^{35}$ R. Landauer, Philos. Mag. 21, 863 (1970). 
${ }^{36}$ M. Büttiker, Y. Imry, R. Landauer and S. Pinhas, Phys. Rev. B 31, 6207 (1985).

${ }^{37}$ D. C. Langreth, Phys. Rev. 150, 516 (1966).

${ }^{38}$ See, for instance, J. Sólyom, Adv. Phys. 28 (1979) 201.

${ }^{39}$ See, for instance, D. L. Cox and A. Zawadowski, Adv. Phys. 47, 599 (1998).

${ }^{40}$ Y. Shimizu, O. Sakai, and S. Suzuki, J. Phys. Soc. Jpn. 67, 2395 (1998).

${ }^{41}$ A. Yeyati, A. Martín-Rodero, and F. Flores, Phys. Rev. Lett. 71, 2991 (1993).

${ }^{42}$ T. Mii and K. Makoshi, Jpn. J. Appl. Phys. 35, 3706 (1996).

${ }^{43}$ O. Takagi and T. Saso, J. Phys. Soc. Jpn. 68, 1997 (1999).

${ }^{44}$ P. L. Pernas, F. Flores, and E. V. Anda, J. Phys. Cond. Matt. 4, 5309 (1992).

${ }^{45}$ Y. Kawahito, H. Kasai, H. Nakanishi, and A. Okiji, J. Appl. Phys. 85, 947 (1999). 


\section{FIGURES}

FIG. 1. Schematic picture of the system

FIG. 2. Schematic picture of the model: $(\bullet)$ interacting region, (०) ideal leads.

FIG. 3. Second-order self-energy $\Sigma_{i j}^{(2)}(i \omega)$.

FIG. 4. Conductance $g_{N}$ as a function of the size $N$ in the inversion symmetric case. Here $v / t=0.8$ and $U /(2 \pi t)=0.4$.

FIG. 5. Conductance for even $N(=2 M)$ as a function of the size $M$. Here $v / t=0.8$, and the repulsion $U /(2 \pi t)$ is taken to be $(\bullet) 0.0,(\boldsymbol{\square}) 0.2,(\bullet) 0.4,(\boldsymbol{\Delta}) 0.6$, and $(\star) 0.8$.

FIG. 6. Conductance vs $U$, for even $N(=2,4,6, \ldots)$. Here $v / t=0.8$ for dashed lines, and $v / t=1.0$ for solid lines. Totally, 20 dashed and 30 solid lines are plotted.

FIG. 7. The ratio $C_{2} / C_{0}$ is plotted for several $v / t(=0.1,0.2, \ldots, 0.9)$ as a function of the size $M$. Here $g_{2 M}=\left(2 e^{2} / h\right)\left[C_{0}-C_{2}\{U /(2 \pi t)\}^{2}+\cdots\right]$.

FIG. 8. The coefficient $C_{4}$ in the case of $v / t=1.0$ is plotted as a function of the size $M$. Here $g_{2 M}=\left(2 e^{2} / h\right)\left[1-C_{4}\{U /(2 \pi t)\}^{2}+\cdots\right]$.

FIG. 9. Conductance vs $U$, for $N=4$ (dashed lines) and $N=40$ (solid lines). Here $v / t$ is taken to be $0.4,0.5, \ldots, 0.9$, and 1.0 .

FIG. 10. Conductance for even $N(=2 M)$ as a function of the size $M$. Here $U /(2 \pi t)=0.3$, and the conductance is normalized by the value for $U=0$, i.e., $g_{2 M}^{(0)} \equiv\left(2 e^{2} / h\right) C_{0}$. The mixing

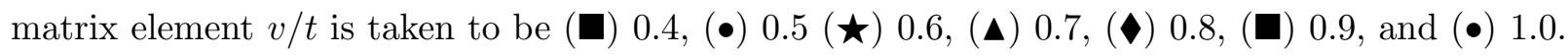

FIG. 11. Conductance $g_{N}$ as a function of the size $N$ in the inversion asymmetric case. Here $v_{L} / t=0.8, v_{R} / t=0.6$, and $U /(2 \pi t)$ is taken to be (a) 0.2 and (b) 0.4 . 
FIG. 12. Conductance vs $U$, for a number of $N(=1,2,3, \ldots, 40)$. Here $v_{L} / t=0.8$ and $v_{R} / t=0.6$

FIG. 13. The coefficient $C_{2}$ as a function of the size $N$. Here $v_{L} / t=0.8, v_{R} / t=0.6$, and $g_{N}=\left(2 e^{2} / h\right)\left[C_{0}-C_{2}\{U /(2 \pi t)\}^{2}+\cdots\right]$. 


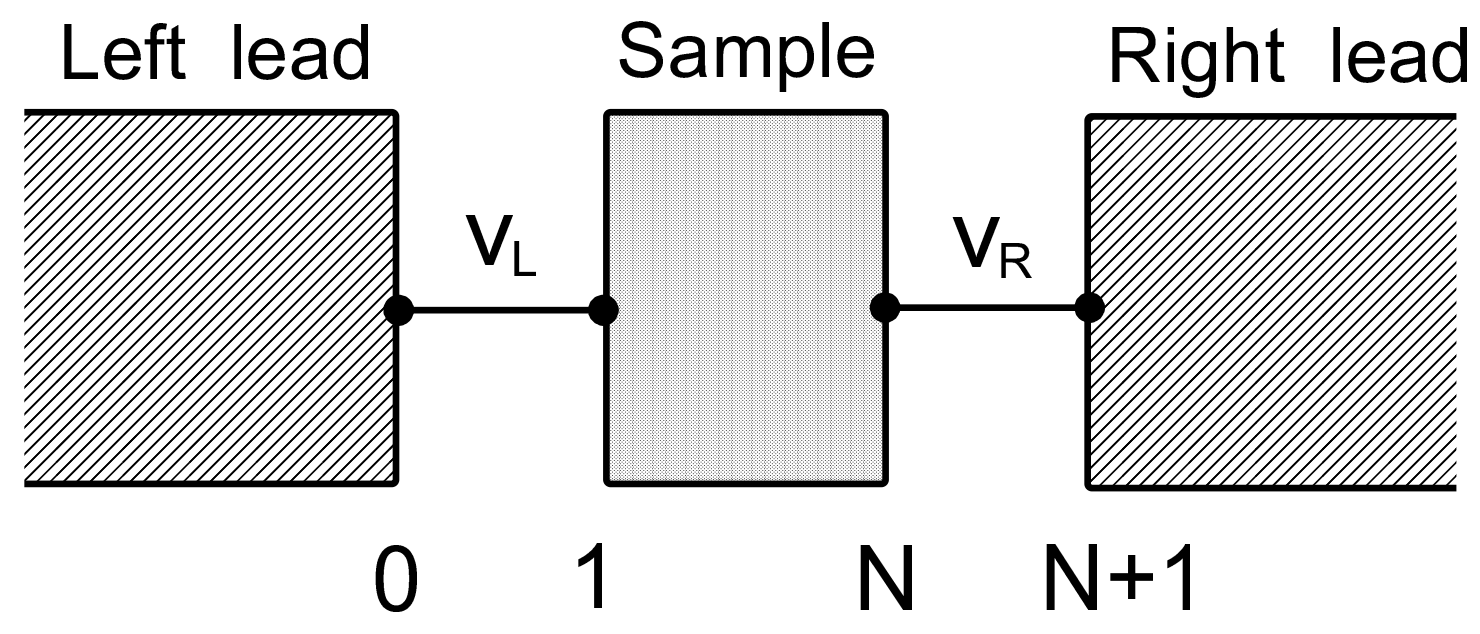




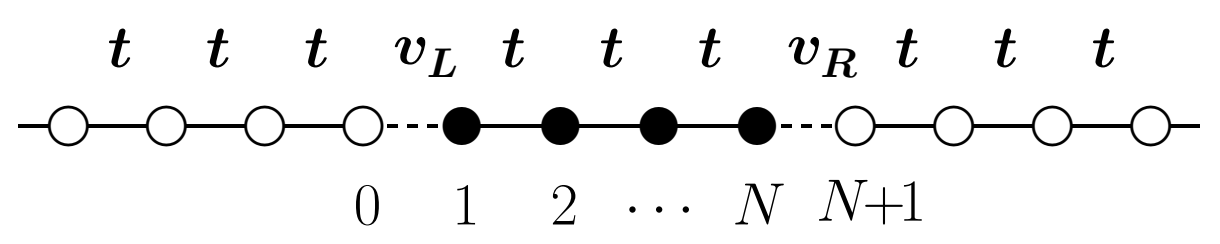




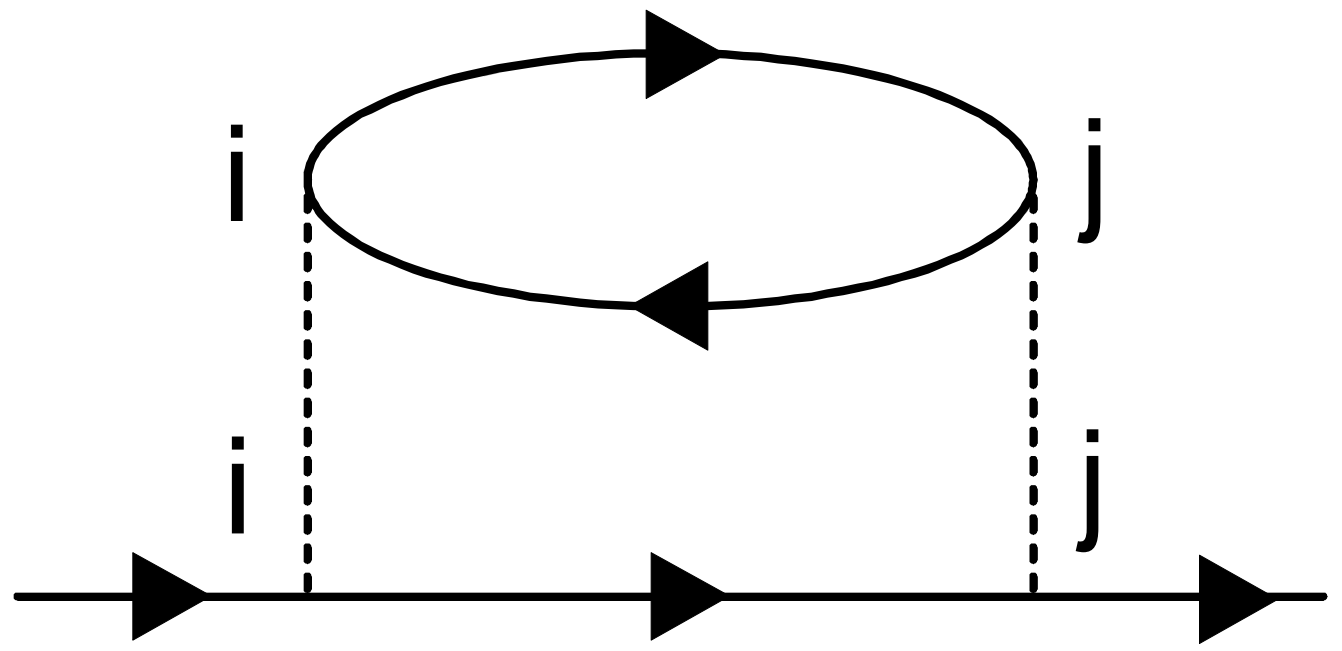




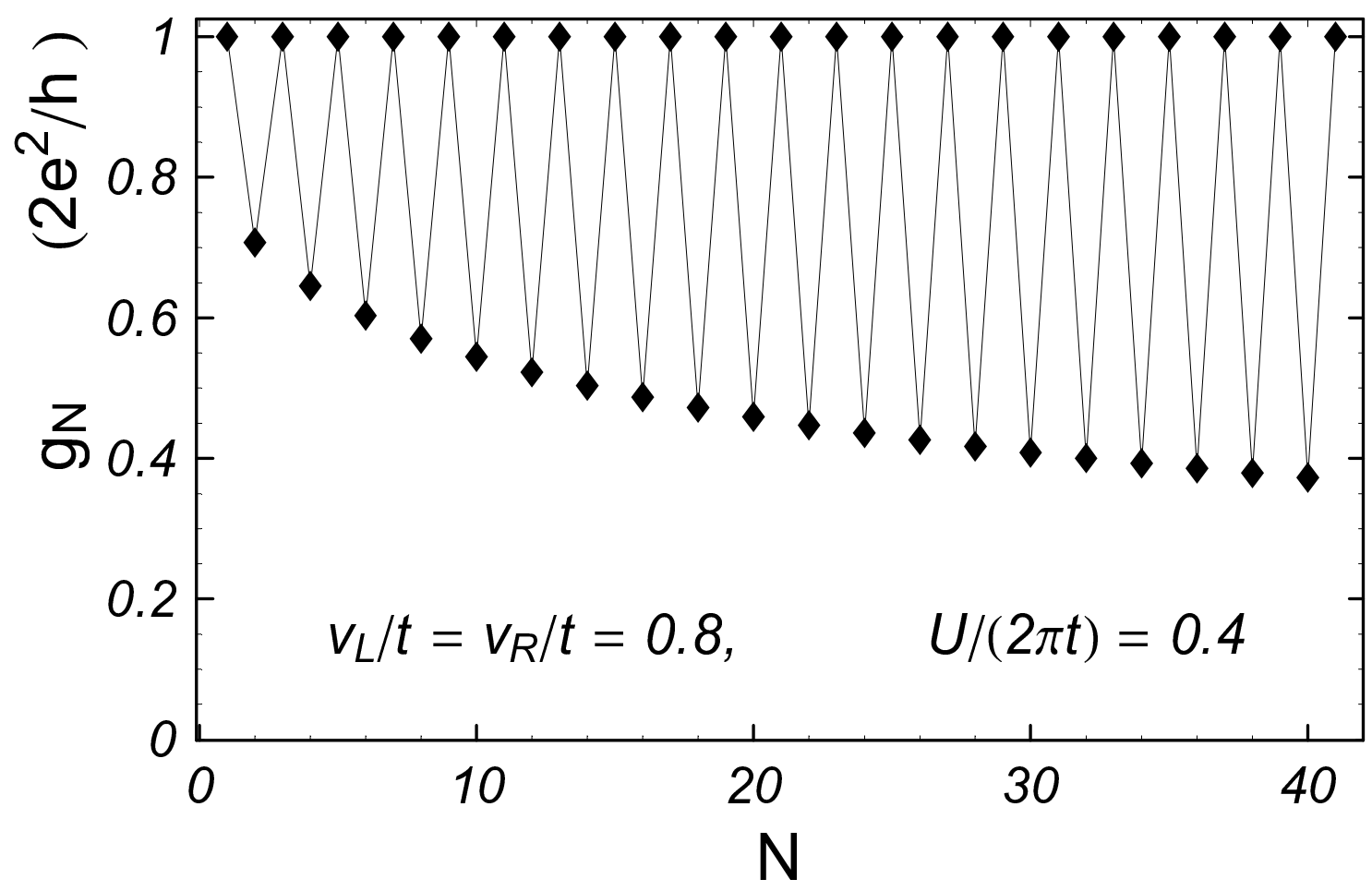




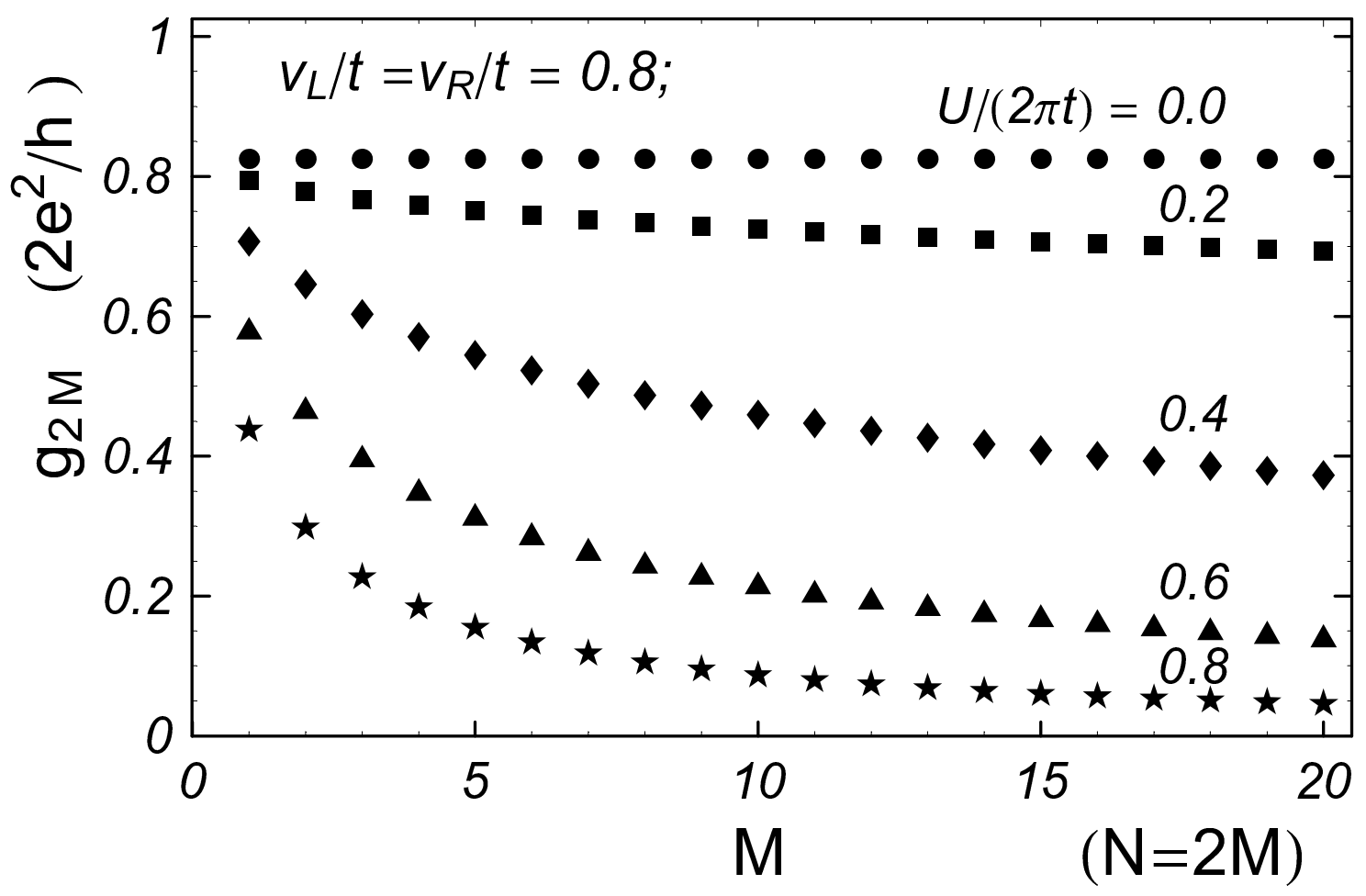




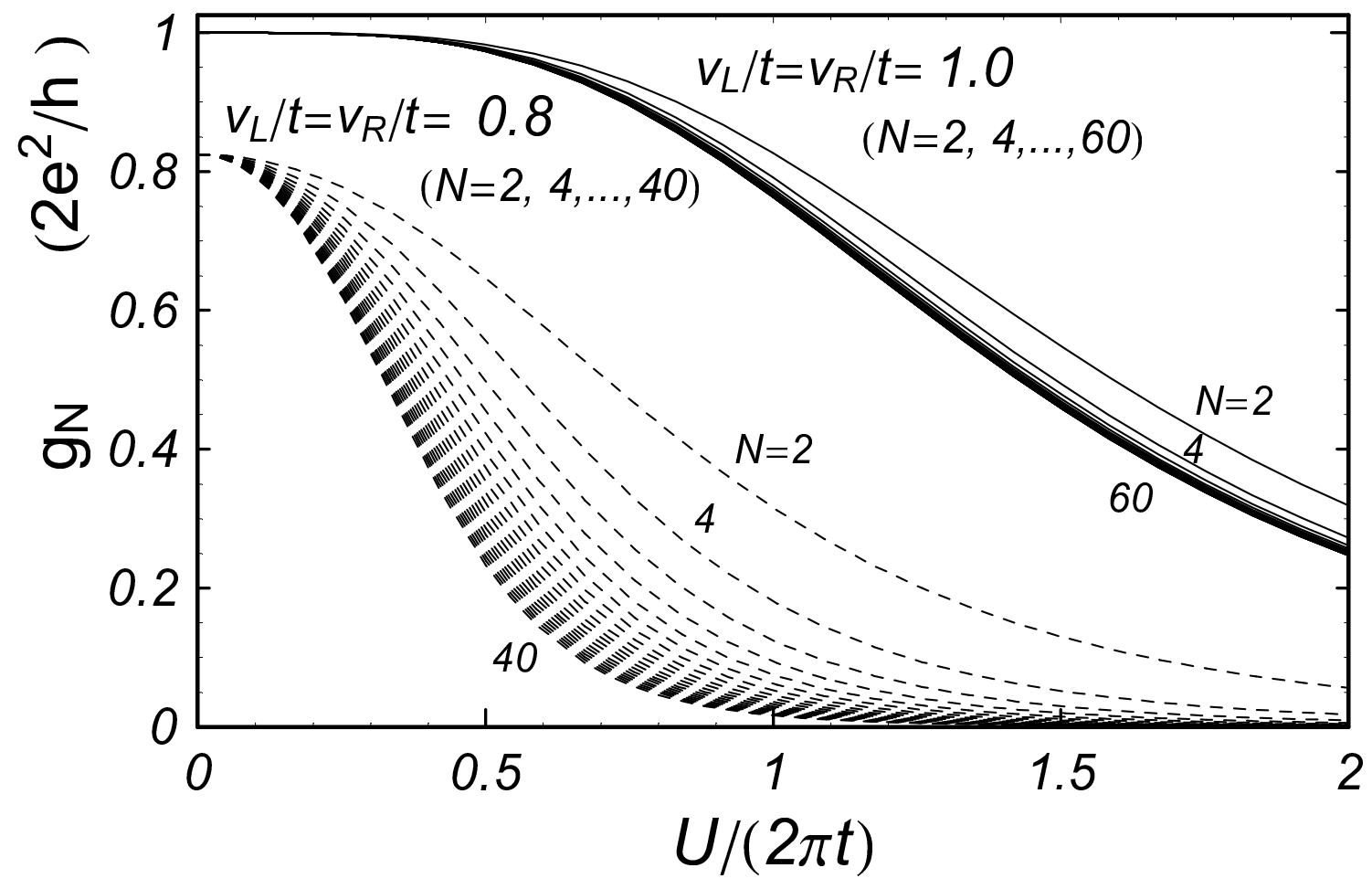




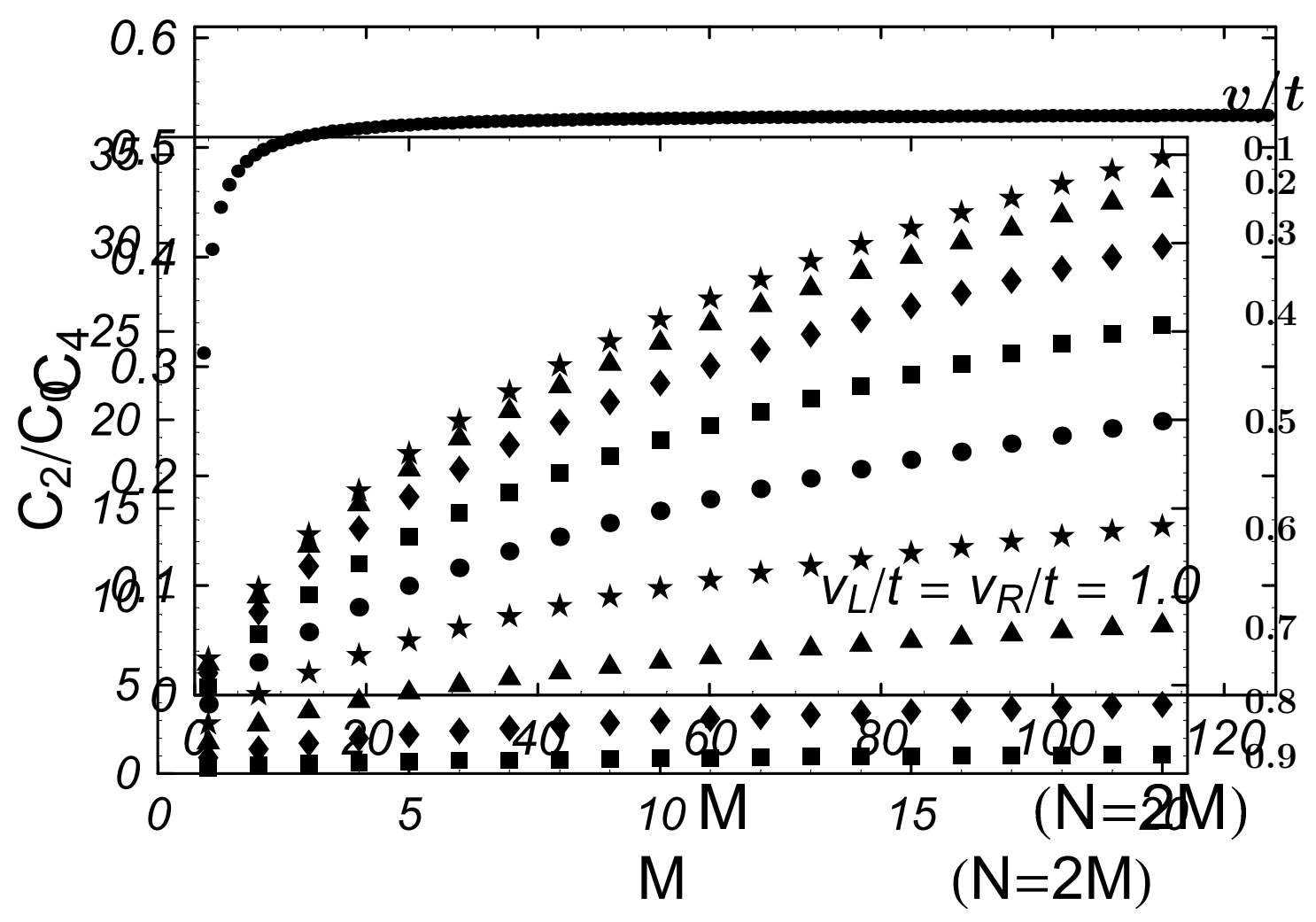




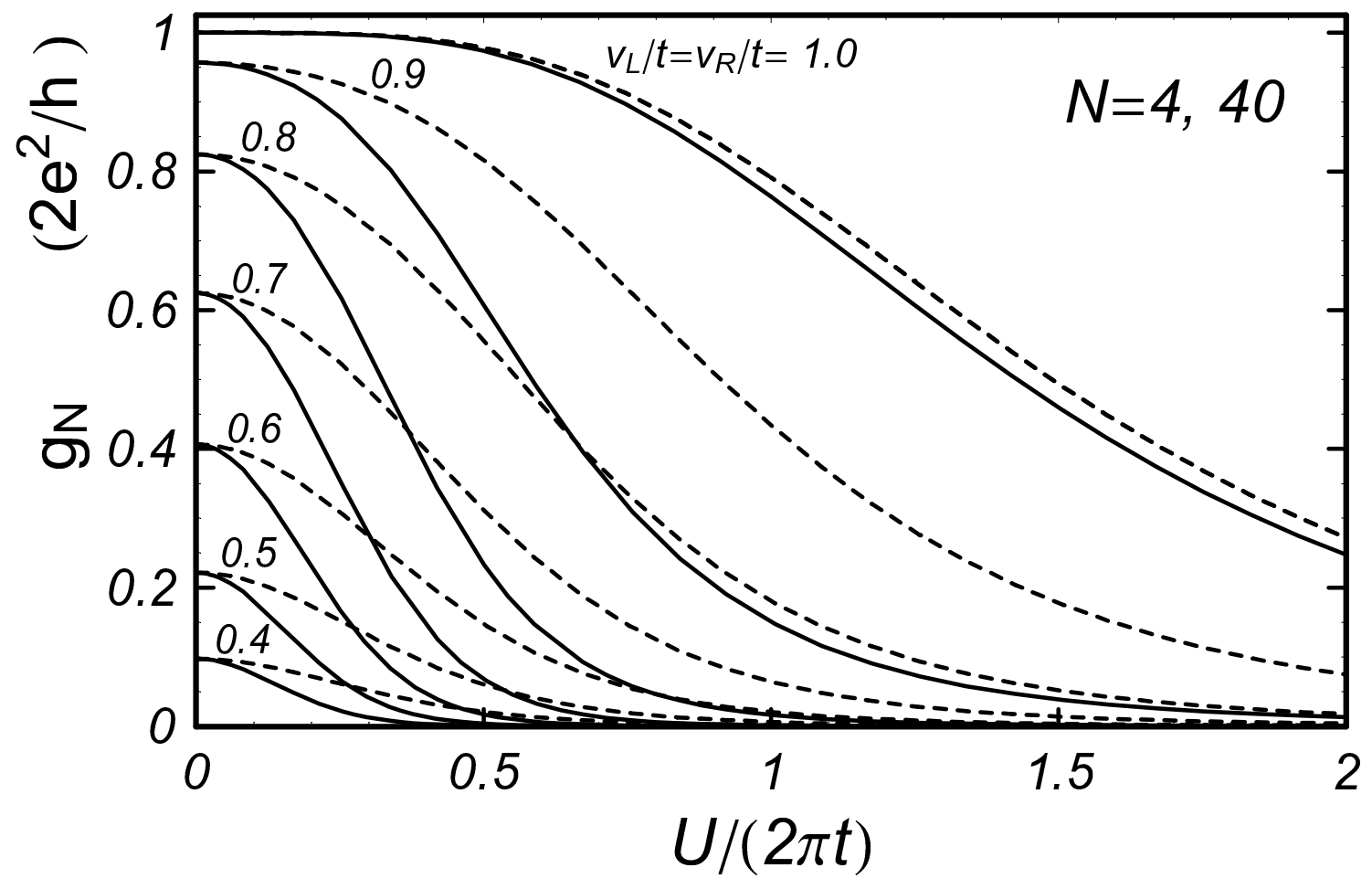




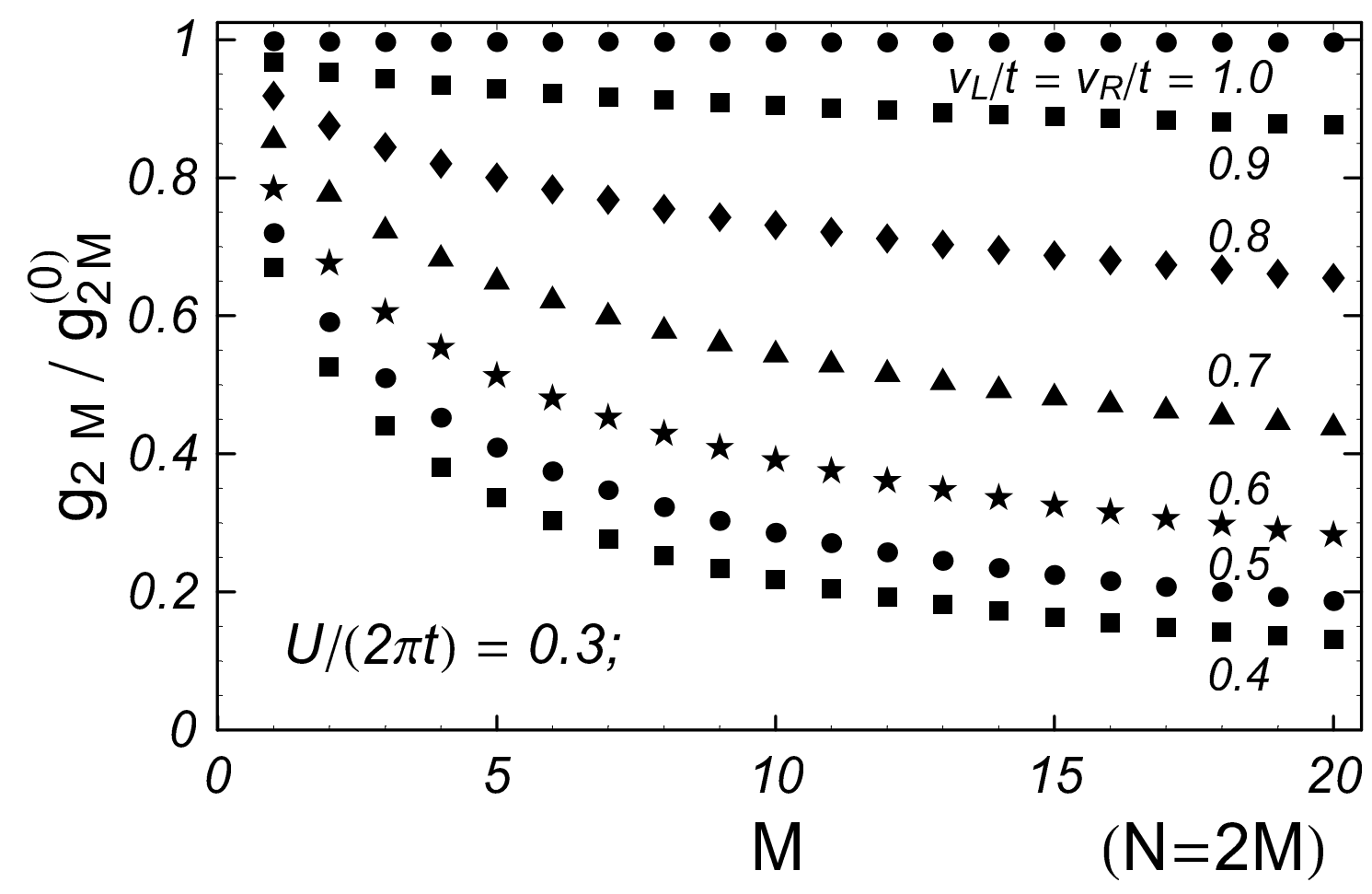




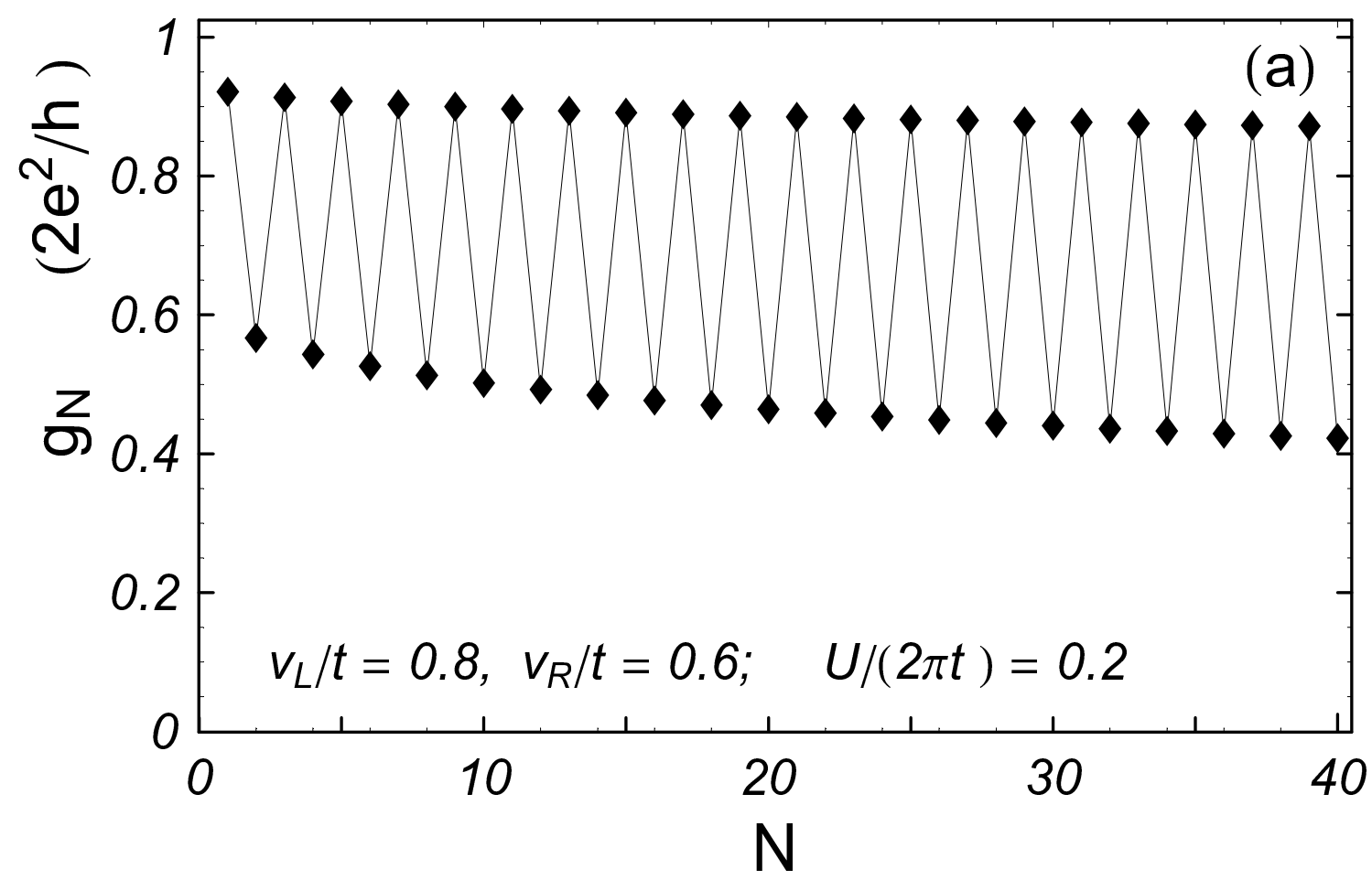




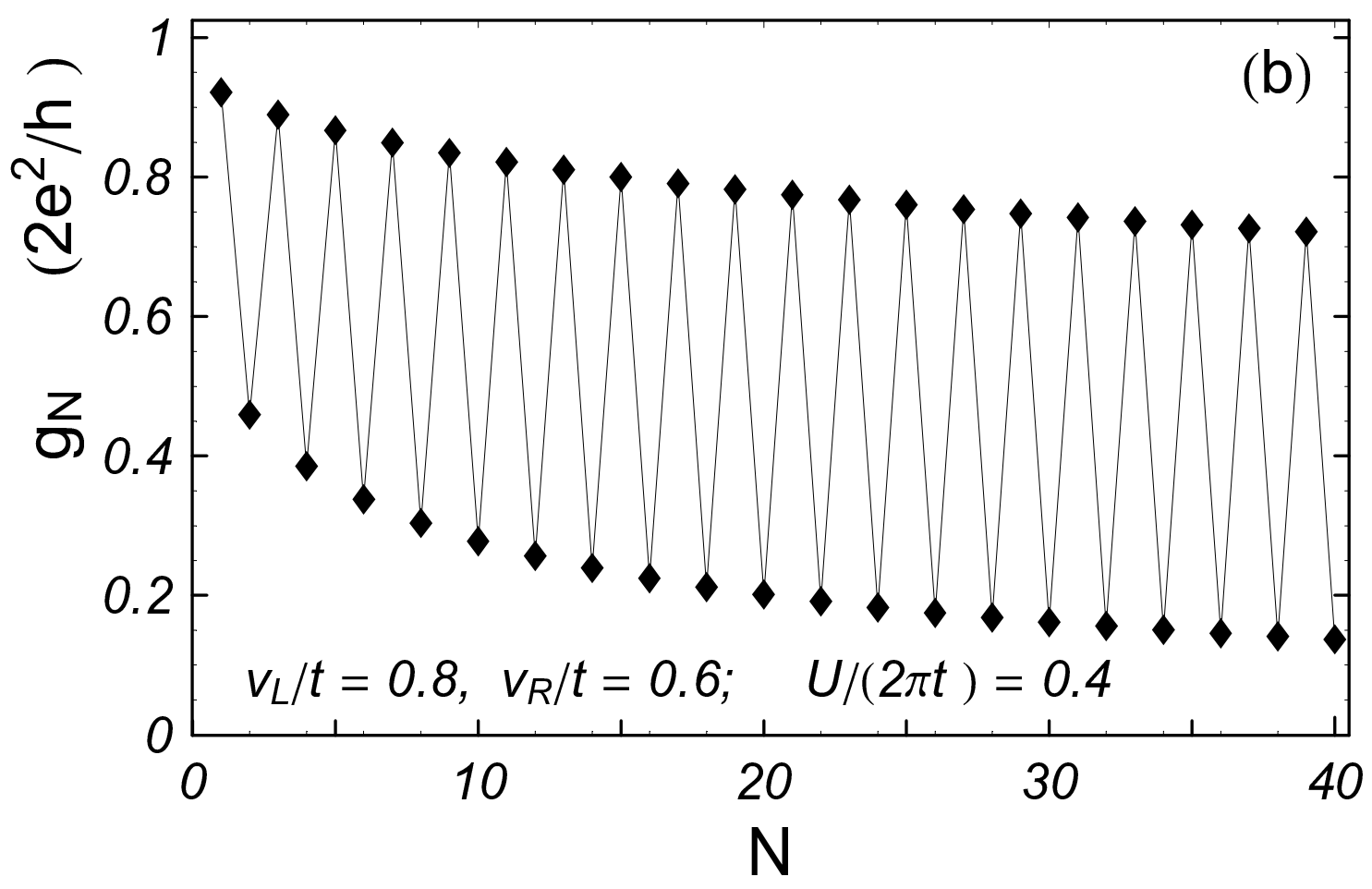




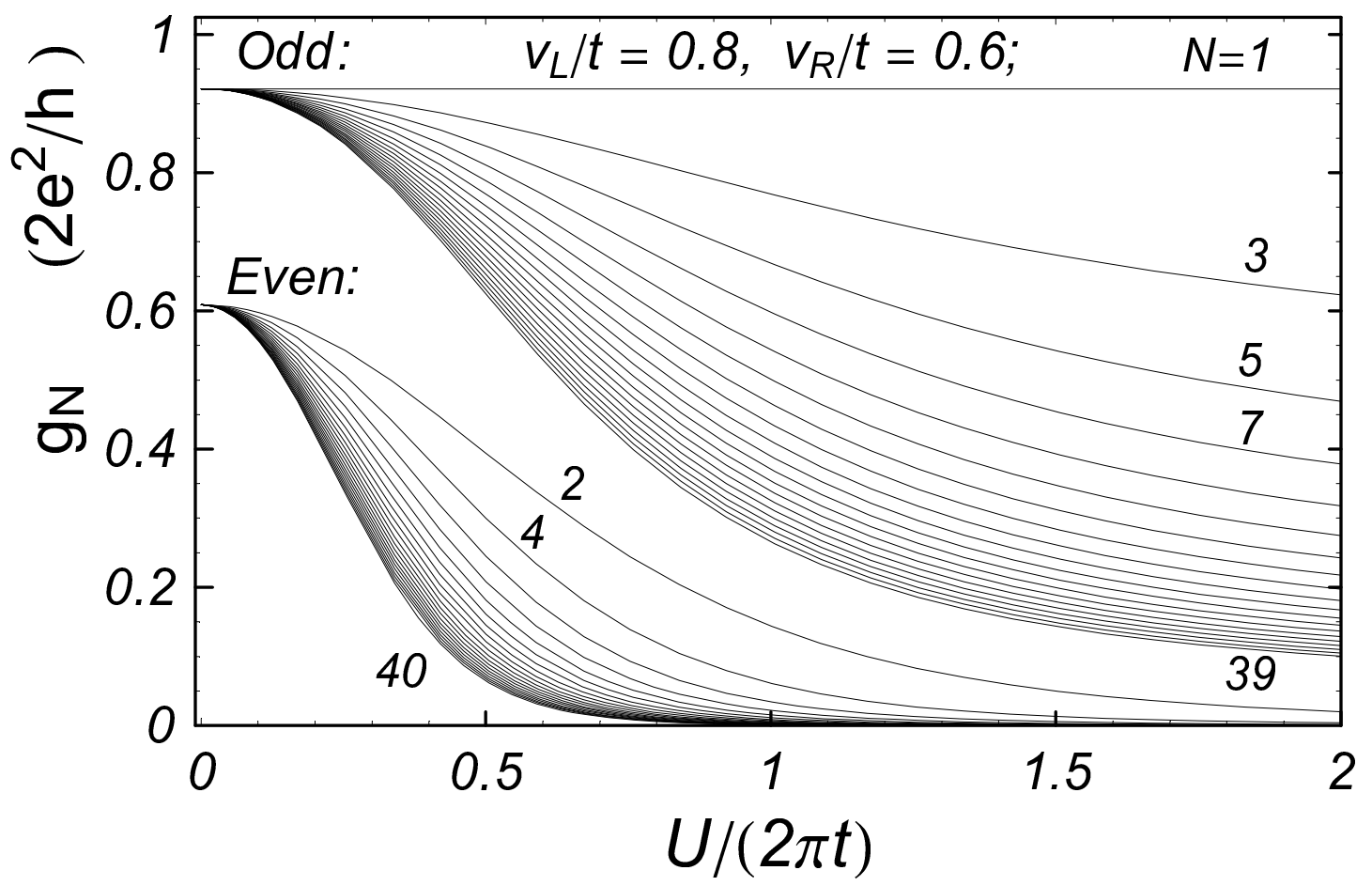




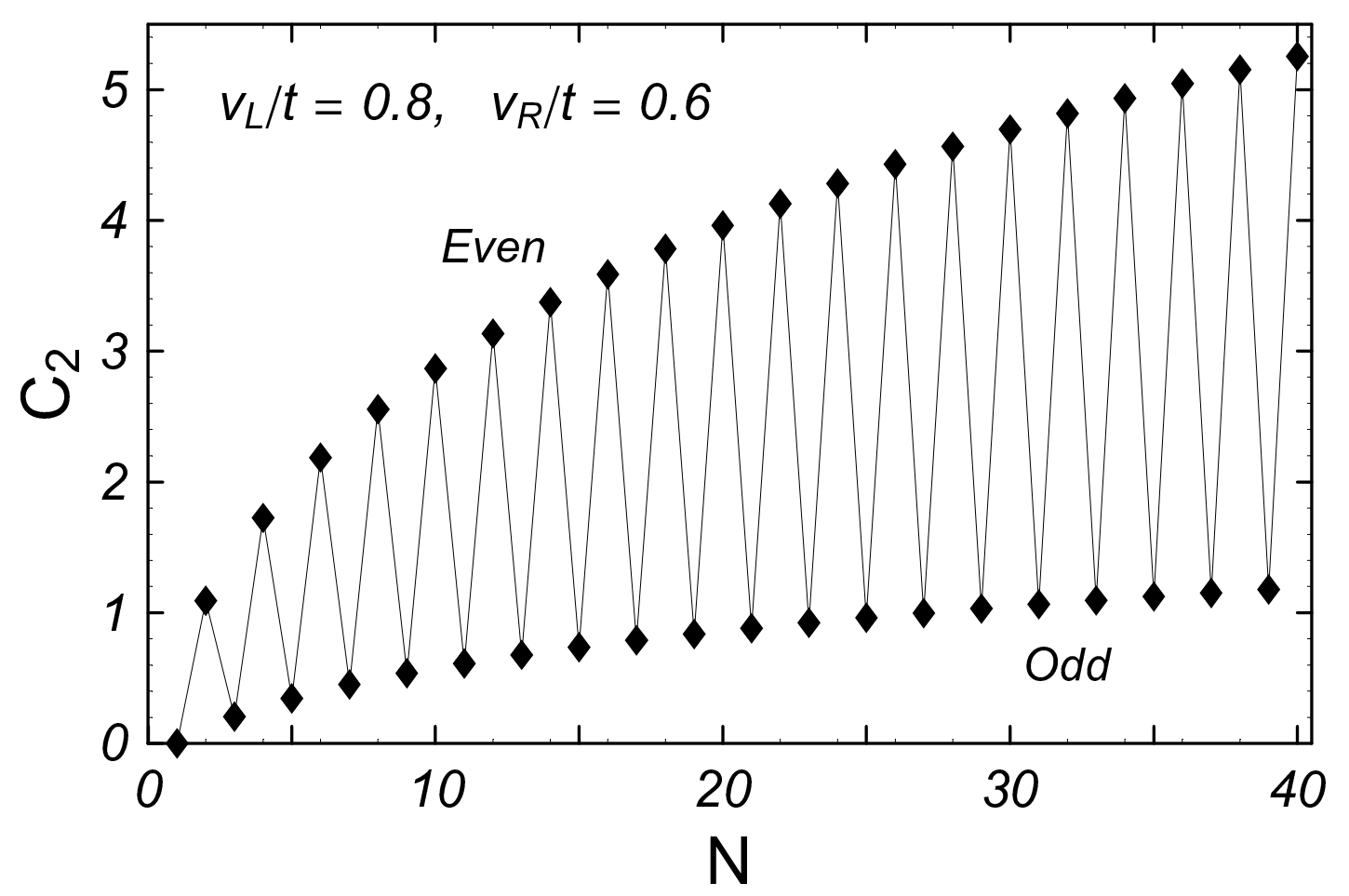

